\title{
Field Dependence of Magnetic Disorder in Nanoparticles
}

\author{
Dominika Zákutnáø, ${ }^{1,2, \$}$ Daniel Nižňanský, ${ }^{3, *}$ Lester C. Barnsley ${ }^{4,8}$ Earl Babcock, ${ }^{4}$ Zahir Salhi, ${ }^{4}$ \\ Artem Feoktystov $\odot,{ }^{4}$ Dirk Honecker $\odot,{ }^{1, \|}$ and Sabrina Disch $\odot^{2, \uparrow}$ \\ ${ }_{1}^{1}$ Institut Laue-Langevin, 71 Avenue des Martyrs, F-38042 Grenoble, France \\ ${ }^{2}$ Department für Chemie, Universität zu Köln, Greinstrasse 4-6, 50939 Köln, Germany \\ ${ }^{3}$ Department of Inorganic Chemistry, Faculty of Science, Charles University in Prague, \\ Hlavova 2030/8, 12843 Prague 2, Czech Republic \\ ${ }^{4}$ Forschungszentrum Jülich GmbH, Jülich Centre for Neutron Science (JCNS) at Heinz Maier-Leibnitz \\ Zentrum (MLZ), Lichtenbergstrasse 1, 85748 Garching, Germany
}

(Received 27 March 2020; revised 18 May 2020; accepted 26 May 2020; published 24 July 2020)

\begin{abstract}
The performance characteristics of magnetic nanoparticles toward application, e.g., in medicine and imaging or as sensors, are directly determined by their magnetization relaxation and total magnetic moment. In the commonly assumed picture, nanoparticles have a constant overall magnetic moment originating from the magnetization of the single-domain particle core surrounded by a surface region hosting spin disorder. In contrast, this work demonstrates the significant increase of the magnetic moment of ferrite nanoparticles with an applied magnetic field. At low magnetic field, the homogeneously magnetized particle core initially coincides in size with the structurally coherent grain of 12.8(2) nm diameter, indicating a strong coupling between magnetic and structural disorder. Applied magnetic fields gradually polarize the uncorrelated, disordered surface spins, resulting in a magnetic volume more than $20 \%$ larger than the structurally coherent core. The intraparticle magnetic disorder energy increases sharply toward the defect-rich surface as established by the field dependence of the magnetization distribution. In consequence, these findings illustrate how the nanoparticle magnetization overcomes structural surface disorder. This new concept of intraparticle magnetization is deployable to other magnetic nanoparticle systems, where the in-depth knowledge of spin disorder and associated magnetic anisotropies are decisive for a rational nanomaterials design.
\end{abstract}

DOI: 10.1103/PhysRevX.10.031019

Subject Areas: Condensed Matter Physics, Magnetism, Nanophysics

\section{INTRODUCTION}

The phenomenon of disorder is ubiquitous in structural science, and different qualities of disorder are evident, ranging from intuitive random disorder to complex types of

\footnotetext{
*Deceased.

${ }^{\dagger}$ Corresponding author. sabrina.disch@uni-koeln.de

*Present address: Department of Inorganic Chemistry, Faculty of Science, Charles University in Prague, Hlavova 2030/8, 12843 Prague 2, Czech Republic.

${ }^{\S}$ Present address: Australian Synchrotron, ANSTO, 800 Blackburn Road, Clayton 3168, Australia.

"Present address: Physics and Materials Science Research Unit, University of Luxembourg, 162A Avenue de la Faïencerie, L-1511 Luxembourg, Luxembourg.

Published by the American Physical Society under the terms of the Creative Commons Attribution 4.0 International license. Further distribution of this work must maintain attribution to the author(s) and the published article's title, journal citation, and DOI.
}

correlated disorder [1]. Correlated disorder is essential for a large number of functional properties, including polar nanoregions in relaxor ferroelectrics [2], colossal magnetoresistance in $\mathrm{La}_{x} \mathrm{Ca}_{(1-2 x)} \mathrm{MnO}_{3}$ [3], the entropic disorder in thermoelectrics [4], and correlated spin disorder leading to quasiparticles such as skyrmions [5] and magnetic monopoles [6]. Being intrinsic to nanomaterials, disorder effects such as surface spin disorder [7] and surface anisotropy $[8,9]$ in magnetic nanoparticles (NPs) crucially determine their magnetization properties including coercivity and superparamagnetism, exchange interactions, and spontaneous magnetization $[10,11]$. These have a pivotal importance for the diverse technological applications of magnetic nanoparticles, such as in recording media [12], biomedicine [13-15], catalysis [16], or battery materials [17]. The impact of disorder on the heating performance of magnetic nanoparticles has recently been demonstrated [18-20]. However, despite the great technological relevance and fundamental importance, the three-dimensional magnetic configuration and the nanoscale distribution of spin disorder within magnetic nanoparticles remain a key challenge. 


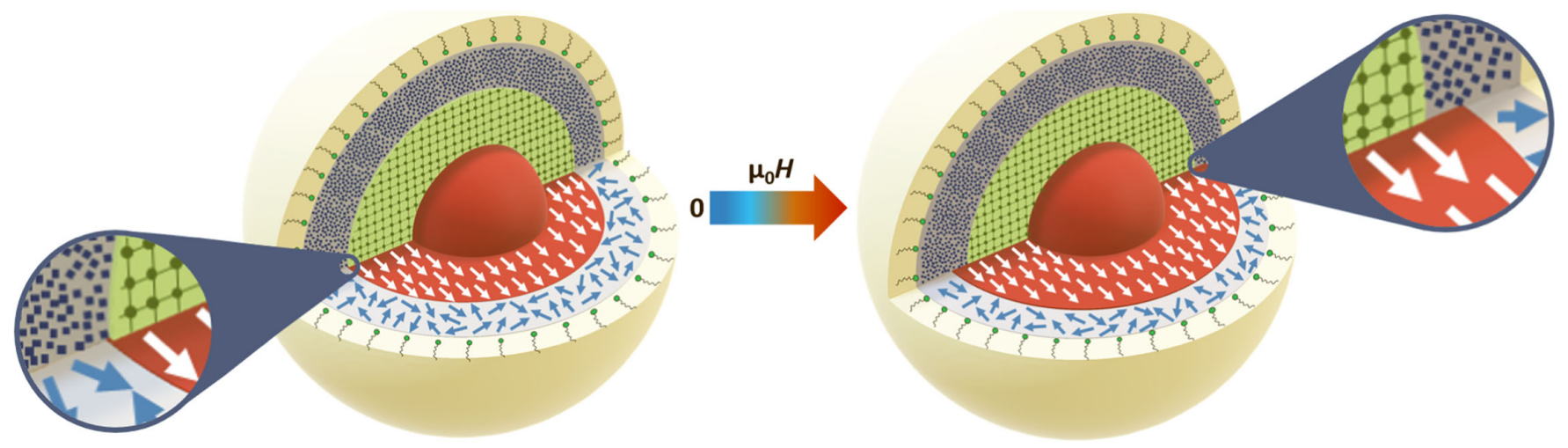

FIG. 1. Schematic of the structural and field-dependent magnetic NP morphology: The vertical cuts represent the structural morphology, consisting of a structurally coherent grain size (green) and structural disorder (blue) within the inorganic particle (gray). The horizontal cuts represent the magnetic morphology, consisting of a collinear magnetic core (red) and spin disorder (blue) within the inorganic particle surface layer (gray). The particle is surrounded by an oleic acid ligand layer (beige). Structural and magnetic particle sizes are equal in zero field (left), whereas the initially disordered surface spins are gradually polarized in the applied magnetic field such that the magnetic radius increases beyond the structurally disordered surface region (right).

Surface spin canting or disorder in magnetic NPs is accessible only indirectly using magnetization measurements, ferromagnetic resonance, Mössbauer spectroscopy [21], x-ray magnetic circular dichroism [22], and electron energy loss spectroscopy [23]. Spin canting at the NP surface arises from low-coordination sites and a high number of broken exchange bonds of the surface atoms [24] and causes a field-dependent shift of the superparamagnetic blocking temperature and exchange bias phenomena [25-27]. Below the spin glass transition, surface spins freeze in a random configuration [28]. In addition, a strong correlation of magnetic and structural disorder is widely accepted [29-33]. In order to reliably discriminate bulk and surface contributions to magnetic disorder, spatial resolution of the intraparticle spin structure is required.

Magnetic small-angle neutron scattering (SANS) is a versatile technique to obtain spatially sensitive information of the spin structure in NPs directly on the relevant nanometer length scale [34]. Using half-polarized SANS (SANSPOL), the quantitative magnetization distribution within maghemite NPs has been resolved, confirming the presence of spin disorder at the particle surface but at the same time revealing a significant degree of spin disorder in the entire NP [35]. Applying SANS with uniaxial polarization analysis (POLARIS) to NP assemblies, a canted magnetic surface shell is reported [36,37], and the temperature dependence of the spin canting in $\mathrm{CoFe}_{2} \mathrm{O}_{4}$ NPs is derived [38]. Micromagnetic simulations of isolated magnetic NPs in a nonmagnetic matrix demonstrate how the interplay between various magnetic interactions leads to nonuniform spin structures in NPs, resulting in a strong variation of the magnetic SANS $[39,40]$. In the context of a polarized SANS study on $\mathrm{Fe}_{3} \mathrm{O}_{4} / \mathrm{Mn}$-ferrite core/shell structures, complementary atomistic magnetic simulations considering a drastically reduced exchange coupling between the core and shell spins reveal no remanence for the shell along with a disordered rather than canted surface spin configuration [41]. Hence, surface spins might potentially be susceptible to intermediate fields, analogous to the spin-flop phase observed in bulk antiferromagnetic oxides [42]. Up to now, all studies of the magnetic nanoparticle spin structure relied on a static picture of a constant, field-independent nanoparticle moment.

In this work, we present the field dependence of collinear magnetization and spin disorder in ferrite nanoparticles and derive the associated disorder anisotropy toward the particle surface with spatial resolution. The spontaneous, noncorrelated spin disorder at the particle surface is strongly related to structural surface disorder. Remarkably, we observe that with an increasing magnetic field the collinear magnetic volume overcomes the structurally coherent particle size. In other words, we demonstrate that the commonly assumed static picture of a constant integral nanoparticle moment with surface spin disorder is not sufficient and needs to be replaced by a field-dependent magnetic nanoparticle core size. This main result of our work is illustrated in Fig. 1. From the field dependence of the magnetic particle volume, we further extract the spatial extent of spin disorder and derive the associated disorder energy distribution based on a free energy calculation. Consistent with macroscopic magnetization and supported by micromagnetic simulations, our findings demonstrate the intricate nature of intraparticle disorder anisotropy.

\section{RESULTS AND DISCUSSION}

\section{A. NP structure and morphology}

The precise evaluation of intraparticle morphologies such as magnetization distribution and spin disorder optimally requires monodisperse samples of noninteracting magnetic nanoparticles. We therefore synthesize oleic acid (OA)-capped cobalt ferrite NPs for our study according to 

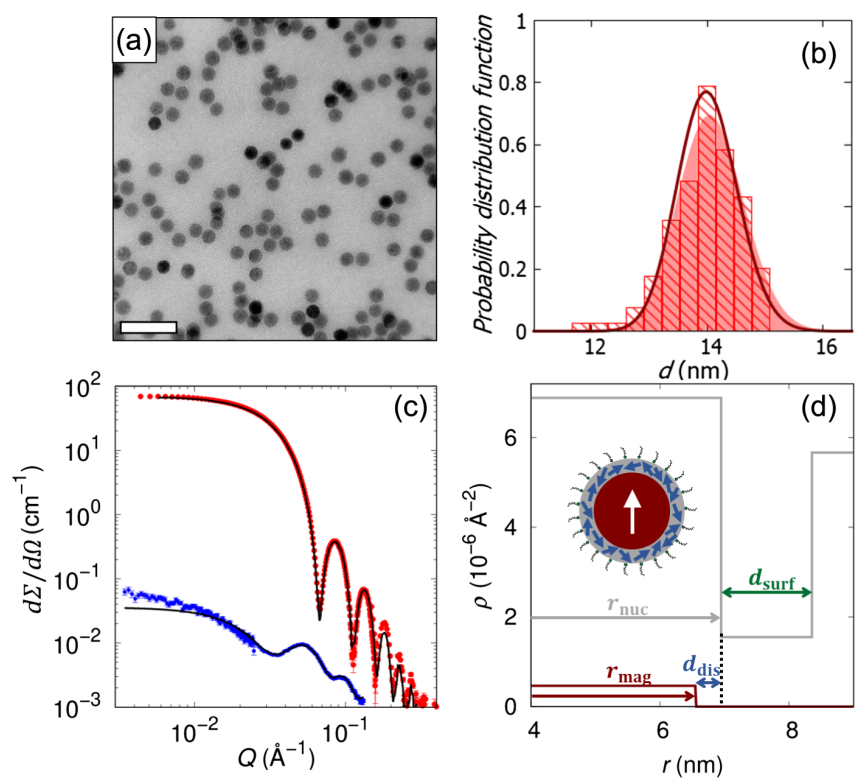

FIG. 2. (a) TEM bright field micrograph (scale bar, $50 \mathrm{~nm}$ ) and (b) particle-size histogram based on the evaluation of 200 particles along with log-normal particle-size distribution obtained from TEM analysis (red surface) and SAXS refinement (line). (c) SAXS (red dots) and nuclear SANS (blue dots) data along with form factor fit (black lines) and (d) radial profiles of the nuclear $\left(\rho_{n}\right.$, gray) and magnetic scattering length densities $\left(\rho_{\text {mag }}\right.$, red). Our model of the magnetic nanoparticle morphology consists of a coherently magnetized particle core with radius $r_{\text {mag }}$ and a magnetically disordered surface shell with thickness $d_{\text {dis }}$ within the inorganic NP with radius $r_{\text {nuc }}$, stabilized by the oleic acid ligand shell with thickness $d_{\text {surf }}$.

Park et al. [43] and stabilize them in the nonpolar solvent toluene [44]. The sample consists of spherical particles with a log-normal size distribution of $3.1(1) \%$ and a mean particle radius of $r_{\text {nuc }}=7.04(5) \mathrm{nm}$ as determined using small-angle $\mathrm{x}$-ray scattering (SAXS), which is in excellent agreement with the results obtained from transmission electron microscopy (TEM) analysis (Fig. 2). These results define the structural parameters of the inorganic particle core size. A Guinier plateau observed in the lower $Q$ range of the SAXS data further demonstrates the absence of interparticle interactions in toluene dispersion [Fig. 2(c)]. The crystal symmetry of the particles determined by powder X-ray diffraction (PXRD) corresponds to the cubic spinel (space group $F d \overline{3} m$ ) with a lattice parameter of $a=8.362(1) \AA$, which is slightly smaller than for bulk $\mathrm{CoFe}_{2} \mathrm{O}_{4} \quad(a=8.3919 \AA)$, an observation commonly reported for nanosized materials [45]. The determined structurally coherent grain size of $d_{\mathrm{XRD}}=12.8(2) \mathrm{nm}$ (Appendix B 1) is significantly smaller than the particle size, indicating structural disorder near the particle surface. An organic ligand shell thickness of $d_{\text {surf }}=1.4(1) \mathrm{nm}$ [Fig. 2(d)] is resolved by the nuclear scattering cross section obtained by SANSPOL [46]. This result is reasonable given the theoretical value of fully stretched OA
$(2.1 \mathrm{~nm})$ and in good agreement with earlier results on OA-stabilized iron oxide NPs in toluene [35]. From the x-ray and neutron scattering length densities of the particle core $\left(\rho_{x}=41.61 \times 10^{-6} \AA^{-2}\right.$ and $\rho_{n}=6.88 \times 10^{-6} \AA^{-2}$, respectively), a Co cation content of 8.4 at.\% is determined according to Végard's law [47]. Assuming neutral overall charge, we consider the formula $\mathrm{Co}_{y} \mathrm{Fe}_{(8-2 y) / 3} \mathrm{O}_{4}$, where $y=1$ represents the cobalt ferrite spinel structure and $y=0$ corresponds to maghemite $\left(\gamma-\mathrm{Fe}_{2} \mathrm{O}_{3}\right)$, and derive a composition of $\mathrm{Co}_{0.22} \mathrm{Fe}_{2.52} \mathrm{O}_{4}$. The stoichiometry is based on Mössbauer spectroscopy measurements (Appendix B 3) demonstrating the absence of $\mathrm{Fe}^{2+}$ in the compound. Energy dispersive $\mathrm{x}$-ray (EDX) scans further support a chemically homogeneous crystalline particle core. A line scan reveals 10 at.\% Co content within the entire particle, whereas an average composition of 9.1 at.\% Co is confirmed by TEM EDX mapping (Fig. 11), both in excellent agreement with the composition derived by small-angle scattering.

\section{B. Field-dependent magnetization distribution}

Using the precise structural particle morphology determined above as a prerequisite, we ascertain the magnetic nanoparticle morphology via the magnetic scattering amplitude of polarized SANS. We model the magnetic nanoparticle morphology with a homogeneously magnetized particle core with radius $r_{\text {mag }}$ and a spin disorder shell of thickness $d_{\text {dis }}$ toward the surface [Fig. 2(d)]. The magnetic particle-size distribution is taken equal to the nuclear size distribution. The in-field or longitudinal magnetization component $M_{z}(H)$ is directly related with the magnetic scattering length density of the particle core $\rho_{\text {mag }}$ determined using polarized SANS [Eq. (B2)]. The nuclear-magnetic interference scattering of our sample [Fig. 3(a)] is consistently described only by a fielddependent variation of both $\rho_{\text {mag }}$ and $r_{\text {mag }}$ [Fig. 3(b)] in contrast to a static model using a field-independent $r_{\text {mag }}$ (Appendix B 7). The magnetic particle radius $r_{\text {mag }}(H)<$ $r_{\text {nuc }}$ increases with the magnetic field, starting from $r_{\text {mag }}\left(H_{\min }\right)=6.3(1) \mathrm{nm}$ at the lowest applied magnetic field of $H_{\min }=11 \mathrm{mT}$ and attaining $r_{\operatorname{mag}}\left(H_{\max }\right)=$ $6.76(4) \mathrm{nm}$ at the highest applied field of $H_{\max }=1.2 \mathrm{~T}$ [Figs. 3(b) and 3(c)]. The spontaneous $r_{\mathrm{mag}}\left(H_{\mathrm{min}}\right)$ is in excellent agreement with the structurally coherent domain size of 12.8(2) nm from PXRD, indicating a structurally homogeneous and spontaneously magnetized particle core smaller than the NP itself. This observation is in line with reports on reduced magnetic domain size in magnetic NPs, suggested by macroscopic magnetization $[31,32,48,49]$ and neutron diffraction [50]. Previous polarized SANS studies indicate spatial correlation of spins near the particle surface giving rise to canted spin structures [36,37]. Simulations propose a variety of different spin canting scenarios, such as collinear, artichoke, throttled, and hedgehog spin structures $[51,52]$. 

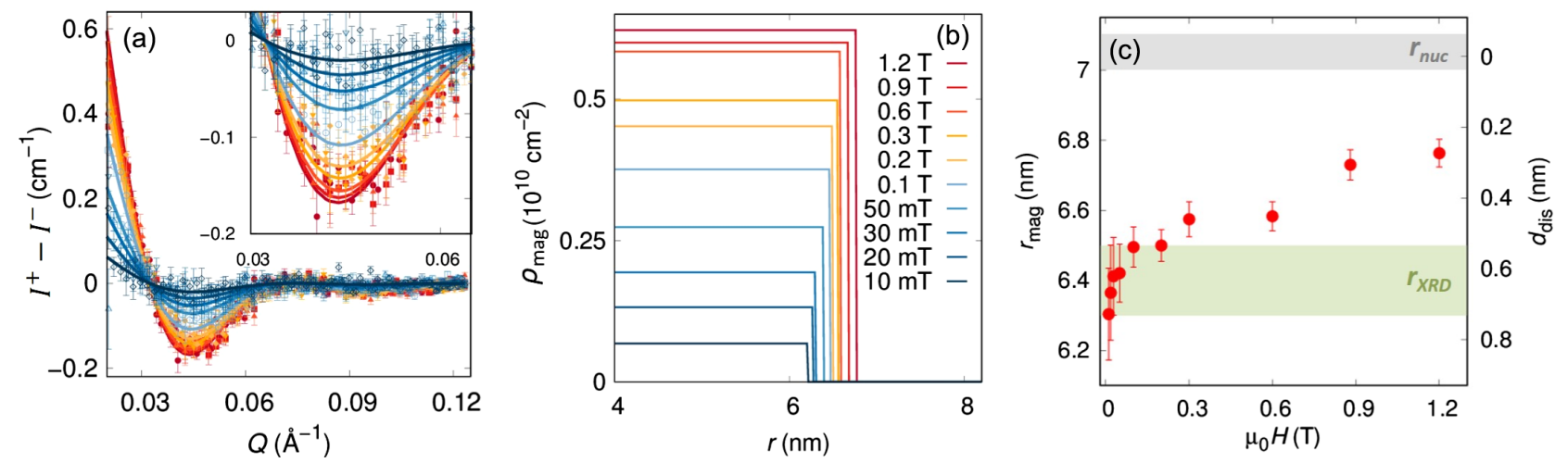

FIG. 3. (a) Nuclear-magnetic scattering interference term $I^{+}-I^{-}$(points) at various applied magnetic fields [same color code as in (b)] and corresponding fits (lines). Inset: Enlarged region of $Q=0.03-0.065 \AA^{-1}$. (b) Field-dependent magnetic scattering length density $\rho_{\text {mag }}$ profiles. (c) Field dependence of the derived magnetic radius $r_{\text {mag }}$ and the disordered shell thickness $d_{\text {dis }}$. The uncertainty intervals of nuclear $\left(r_{\text {nuc }}\right)$ and structurally coherent radius $\left(r_{\mathrm{XRD}}\right)$ are indicated in gray and green, respectively.

To distinguish between correlated (spin canting) and noncorrelated (spin disorder) spins near the NP surface, we perform spin-resolved SANS (POLARIS) on the noninteracting nanoparticles in dispersion (Fig. 4). POLARIS gives access to the Fourier transformation of magnetization correlations along the three Cartesian directions [34]. In our case of spherical nanoparticles, the transversal magnetization correlations $\left|\widetilde{M}_{\perp}\right|^{2}=\left|\widetilde{M}_{x}\right|^{2}=\left|\widetilde{M}_{y}\right|^{2}$ are assumed to be equal for symmetry reasons. Based on POLARIS data of two different applied magnetic fields (Fig. 4), we conclude that the particles do not exhibit a coherently ordered, transversal magnetization component $\left|\widetilde{M}_{\perp}\right|^{2}$. Despite low scattering statistics, in particular, in the spin-flip data, the fit parameters of nuclear scattering amplitude, incoherent background, and longitudinal magnetization obtained from the different datasets are in excellent, consistent agreement, including the expected slight increase of the longitudinal magnetization following the orientation of the particle moment with the applied field (see details in Appendix B 8). The absence of a coherent, elastic scattering contribution originating from transversal magnetization $\left|\widetilde{M}_{\perp}\right|^{2}$ is a strong indication of a noncorrelated, random
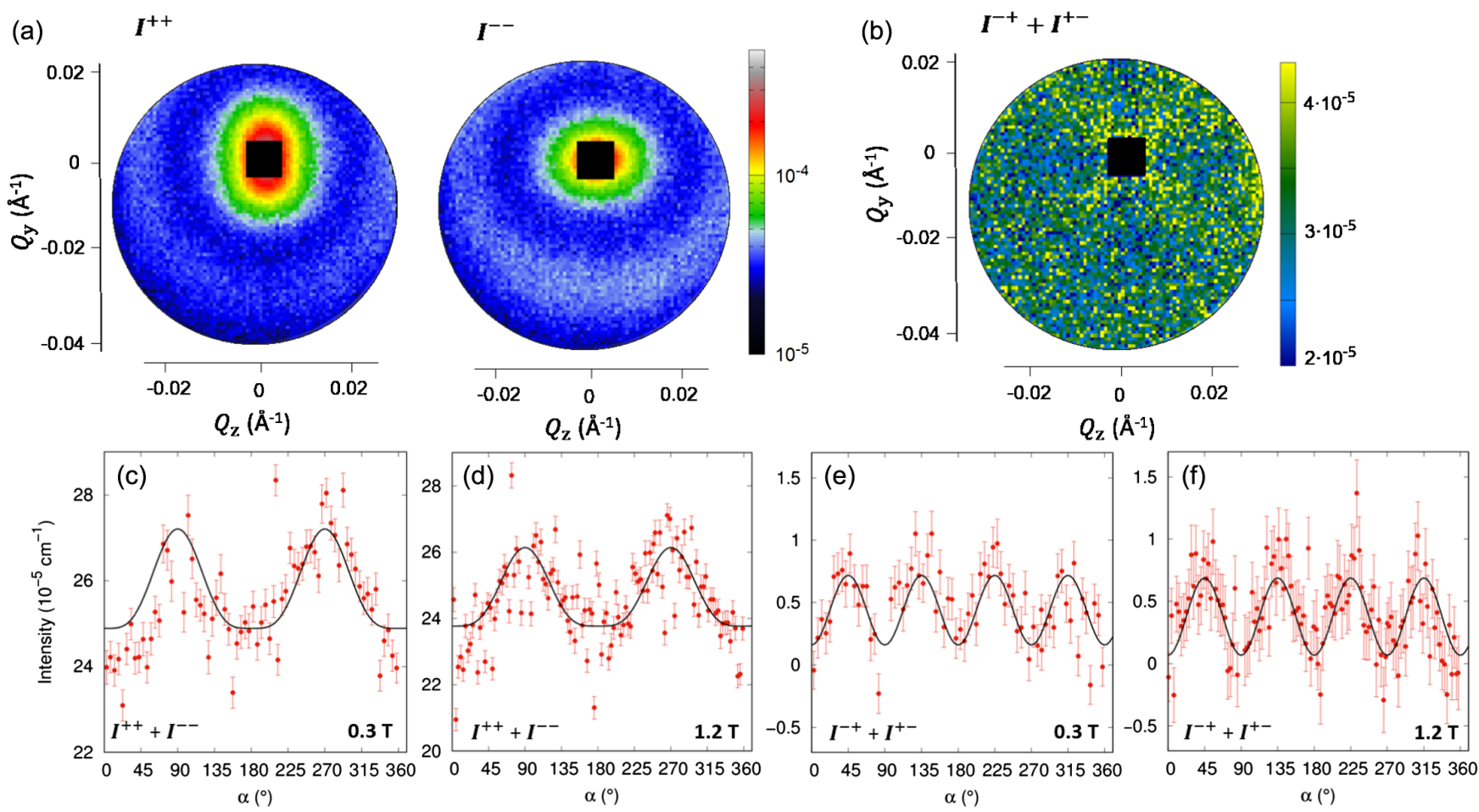

FIG. 4. Spin-resolved SANS (POLARIS) by cobalt ferrite nanoparticles: (a) non-spin-flip (NSF) $\left(I^{++}, I^{--}\right.$) and (b) averaged spin-flip (SF) $\left[\left(I^{+-}+I^{-+}\right) / 2\right] 2 \mathrm{D}$ scattering cross sections at an applied magnetic field of $1.2 \mathrm{~T}$. (c)-(f) NSF and SF azimuthal scattering intensities, radially averaged in a $Q$ range of $0.006-0.016 \AA^{-1}$, recorded at 0.3 and $1.2 \mathrm{~T}$ with corresponding fits (black line). 
spin disorder for our sample, in contrast to the canted spin structures suggested in the literature.

Whereas the existence of surface spin disorder and canting has been under debate in the past, the field-induced reduction of the magnetically disordered surface shell thickness $d_{\text {dis }}(H)=r_{\text {nuc }}-r_{\text {mag }}(H)$ [Fig. 3(c)] revealed in this work is an entirely new observation. At the lowest applied magnetic field of $11 \mathrm{mT}, 28(5) \%$ of the particle volume is associated to a disordered surface with a thickness of $d_{\text {dis }}=0.7(1) \mathrm{nm}$. The coherently magnetized particle core size, and, hence, the magnetic particle moment, gradually increases with the applied magnetic field, indicating a field-induced alignment of the initially disordered spins even beyond the structurally coherent grain size. At maximum applied field $\left(\mu_{0} H_{\max }=1.2 \mathrm{~T}\right)$, a nonmagnetic surface layer of $d_{\text {dis }}=0.28(6) \mathrm{nm}$ persists, implying a strong degree of spin disorder in $12(2) \%$ of the particle volume that cannot be overcome by the magnetic field applied in this study.

The spatially resolved magnetization obtained using SANSPOL gives unprecedented detailed insight into the spontaneous nanoparticle magnetization as a valuable complement to standard macroscopic magnetization measurements. In the conventional picture, the isothermal magnetization for a superparamagnet is described based on the assumption of a field-independent, constant magnetic particle moment. The relative magnetization is described as

$$
\frac{\langle M\rangle}{M_{S}}=\langle\cos \gamma(H)\rangle=L(\xi)=\operatorname{coth} \xi-\frac{1}{\xi},
$$

where $\langle\cos \gamma(H)\rangle$ is the orientation average over the particle ensemble, with the angle $\gamma$ between the magnetic moment of a particle and the applied magnetic field $\mathbf{H}$. The Langevin parameter is given as $\xi=\mu \mu_{0} H / k_{B} T$ with $\mu_{0}$ the permeability of free space, $\mu$ the integrated particle moment, $k_{B}$ denoting the Boltzmann constant, and $T$ the temperature. The macroscopic volume magnetization $\left\langle M_{\mathrm{VSM}}\right\rangle$ is typically set in relation with the entire sample (and nanoparticle) volume, i.e., disregarding the potentially reduced magnetic volume due to a spin disordered surface region. A Langevin fit of $\left\langle M_{\mathrm{VSM}}\right\rangle$ obtained at $300 \mathrm{~K}$ (Fig. 5) yields a particle moment of $\mu=1.2(1) \times 10^{4} \mu_{B}$ with a spontaneous magnetization $M_{S, \mathrm{VSM}}=135(2) \mathrm{kA} / \mathrm{m}$. The derived spontaneous magnetization is significantly smaller than for bulk cobalt ferrite $(400 \mathrm{kA} / \mathrm{m})$ [53]. In addition, an excess paramagnetic susceptibility of $\chi_{\mathrm{VSM}}=6.33(6) \times 10^{-2}$ is evident from the nonsaturating magnetization at high applied field. Such excess paramagnetic susceptibility along with reduced spontaneous magnetization as compared to the bulk material is commonly associated to the presence of disordered, misaligned moments [7] in addition to the linear highfield susceptibility originating from canted sublattice spins in the bulk material [54]. The estimated magnetic particle volume $V_{\text {mag, } \mathrm{VSM}}=\mu / M_{S, \mathrm{VSM}}=8.3(2) \times 10^{-25} \mathrm{~m}^{3}$

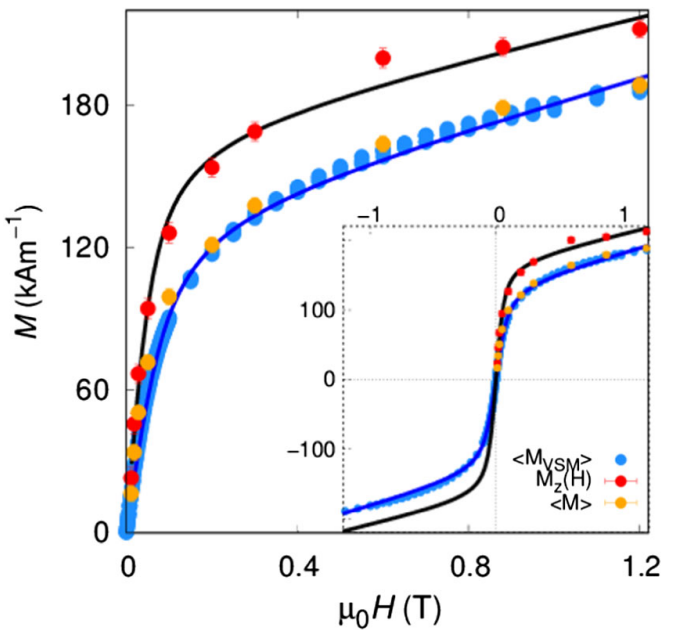

FIG. 5. Macroscopic longitudinal magnetization $\left(\left\langle M_{\mathrm{VSM}}\right\rangle\right)$ measured at room temperature in comparison with the longitudinal particle core magnetization $\left[M_{z}(H)\right]$ and the particle volume averaged magnetization $(\langle M\rangle)$, both derived from SANSPOL. Corresponding fits are shown as lines. Inset: Full data range for $\left\langle M_{\mathrm{VSM}}\right\rangle$.

is comparable to the magnetic particle volume $V_{\text {mag,SANS }}=$ $1.05(6) \times 10^{-24} \mathrm{~m}^{3}$ derived from the minimal magnetic radius at the lowest applied field. Both magnetic particle volumes are considerably smaller than the morphological NP volume $V_{\text {nuc }}=\frac{4}{3} \pi r_{\text {nuc }}^{3}=1.46(3) \times 10^{-24} \mathrm{~m}^{3}$ derived from SAXS. This discrepancy is commonly attributed to surface disorder effects.

The longitudinal magnetization $M_{z}(H)$ is based on the coherently magnetized particle core and, thus, takes into account the variation of the magnetic particle volume. Application of the same Langevin fit as above reveals an enhanced magnetization response (red dots in Fig. 5). We extract a spontaneous magnetization $M_{S \text {,core }}=$ $170(7) \mathrm{kA} / \mathrm{m}$, which is larger than $M_{S, \text { VSM }}$ but still substantially smaller than the bulk saturation magnetization of cobalt ferrite. The coherently magnetized particle core contributes a particle moment of $\mu=1.8(2) \times 10^{4} \mu_{B}$ that yields a magnetic particle volume $V_{\text {mag,core }}=\mu / M_{S \text {,core }}=$ $1.0(1) \times 10^{-24} \mathrm{~m}^{3}$, in excellent agreement with $V_{\mathrm{mag}, \text { SANS }}$. We further determine an excess paramagnetic susceptibility of $\chi_{\text {core }}=5(1) \times 10^{-2}$ that is slightly reduced as compared to $\chi_{\mathrm{VSM}}$. Our spatially resolved approach thus reveals a homogeneously magnetized particle core with larger magnetization and less spin disorder than expected based on only the macroscopic measurements but still far from bulk $\mathrm{CoFe}_{2} \mathrm{O}_{4}$ characteristics.

Whereas effects such as spin disorder or sublattice spin canting are commonly parametrized by a linear high-field susceptibility, this simple approach bears the risk to overcompensate further delicate sample-related phenomena such as a bimodal distribution of the particle moment [55] or the field dependence of $\mu(H)$ that we observe using 
polarized SANS. A closer look into Fig. 5 reveals signatures of such discrepancies as systematic variations between the fit and the experimental data. Numerical inversion methods for data refinement exist that allow one to determine the moment distribution without a priori assumptions on a functional form and, hence, enable the detection of finer details on the structural and magnetic characteristics of magnetic nanoparticles not retrieved by standard model fits [55-58]. In our case, a model-free analysis of the underlying moment distribution indicates the presence of at least two distinct features which we attribute to the core moments and to the shell magnetization (Appendix B 2). The actual field dependence of the particle moment, however, cannot be resolved from macroscopic magnetization data alone and requires a spatially sensitive technique such as polarized SANS.

As a consistency proof, we relate the longitudinal magnetization $M_{z}(H)$ to the average magnetization of the inorganic particle volume according to $\langle M\rangle=$ $M_{z}(H) V_{\text {mag }}(H) / V_{\text {nuc }}$ (orange dots in Fig. 5). The good agreement with the integral magnetization confirms the reliability of the refinement for a coherently magnetized core with a magnetically disordered surface shell that is further supported by our POLARIS analysis. In consequence, the observed low NP magnetization as compared to the bulk material is a result of both surface spin disorder and reduced magnetization related to a combined effect of the nonstoichiometric amount of $\mathrm{Co}$ in the material and structural disorder within the coherently magnetized particle core. For a composition of $\mathrm{Co}_{0.5} \mathrm{Fe}_{2.5} \mathrm{O}_{4}$, a $50 \%$ reduced saturation magnetization compared to nominal $\mathrm{CoFe}_{2} \mathrm{O}_{4}$ is reported [59,60]. For our sample with a composition of $\mathrm{Co}_{0.22} \mathrm{Fe}_{2.52} \mathrm{O}_{4}$, a significant decrease in $M_{S}$ may thus be expected. In addition, high-resolution TEM (HRTEM) indicates structural disorder in the NP interior including dislocations in the (220) lattice plane (Fig. 6). Such structural disorder has been observed before in maghemite spinel NPs [30,33] and is likely correlated with intraparticle spin disorder leading to reduced spontaneous magnetization as well as excess paramagnetic susceptibility in the coherently magnetized NP core. A detailed investigation of the defected internal structure of small iron oxide nanoparticles has recently revealed uncompensated spin density at atomic-scale interfaces as a result of noncubic local symmetry, in line with enhanced spin canting in the particle interior [20].

\section{Micromagnetic approach}

The uncovered field dependence of the magnetic radius may originate in either intrinsic magnetic phenomena, such as surface anisotropy, or structural fluctuations, such as gradual lattice distortions near the particle surface. We therefore apply a micromagnetic approach in terms of Ginzburg-Landau theory as introduced by Kronmüller and Fähnle [61] to describe the magnetic scattering

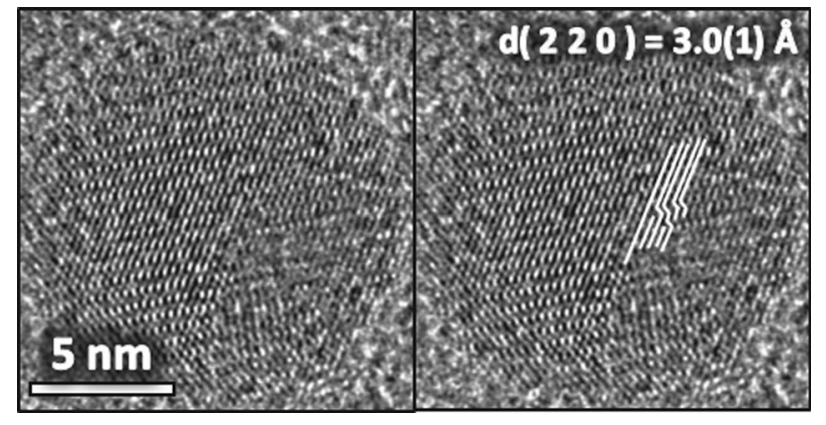

FIG. 6. HRTEM micrographs of one representative NP with visible dislocation.

amplitude under the influence of spatially random magnetocrystalline and magnetostrictive fluctuations (Appendix C). The refinement (Fig. 14) based on a core-shell structure for the magnetic perturbation fields converges for an inner anisotropy constant $K_{\text {in }}=H_{K \text {,in }} \cdot M_{S}=86(52) \mathrm{kJ} / \mathrm{m}^{3}$, suggesting a significant amount of magnetic disorder in the particle core interior. Further relevant parameters obtained include an outer anisotropy $K_{\text {shell }}=241(91) \mathrm{kJ} / \mathrm{m}^{3}$, a shell thickness $d_{\text {dis }}=1.3(2) \mathrm{nm}$, and a spontaneous magnetization of $M_{S}=245(19) \mathrm{kA} / \mathrm{m}$. The derived spontaneous magnetization and shell thickness are in good agreement with the spontaneous magnetization in the particle core and the initial disorder shell thickness determined by SANSPOL. The mean anisotropy field inside the particle $\left\langle H_{K}\right\rangle=0.6(2) \mathrm{T}$ corresponds to a magnetocrystalline anisotropy constant of $\left\langle K_{b}\right\rangle=149(56) \mathrm{kJ} / \mathrm{m}^{3}$, which can be considered as an average value over the entire particle and is in the range of anisotropy constants reported for $\mathrm{CoFe}_{2} \mathrm{O}_{4}$ [62]. This result indicates that fluctuations of magnetic parameters, i.e., magnetocrystalline anisotropy and magnetostrictive contributions, are the most likely sources of the variation of the magnetic radius with the field. In the following, we consider the magnetic field energy associated to the field-dependent variation of the magnetic volume to extract more detailed, model-independent, and spatially resolved information on the extent and strength of the microstructural fluctuations.

\section{Spatially resolved disorder energy}

The field-dependent increase of the magnetic volume and the corresponding magnetic field energy occur in excess of disorder energy that has to be overcome to polarize the initially disordered surface spins (Appendix D). The free energy with respect to the initial volume of the magnetic core is given as

$$
E_{\mathrm{dis}}(H)=\mu_{0} H M_{z}(H)\left[V_{\mathrm{mag}}(H)-V_{\mathrm{mag}}\left(H_{\mathrm{min}}\right)\right] .
$$

The gradual growth of the magnetic volume with an increasing field is a consequence of a distribution of spin disorder energies such that the spin system is harder to 

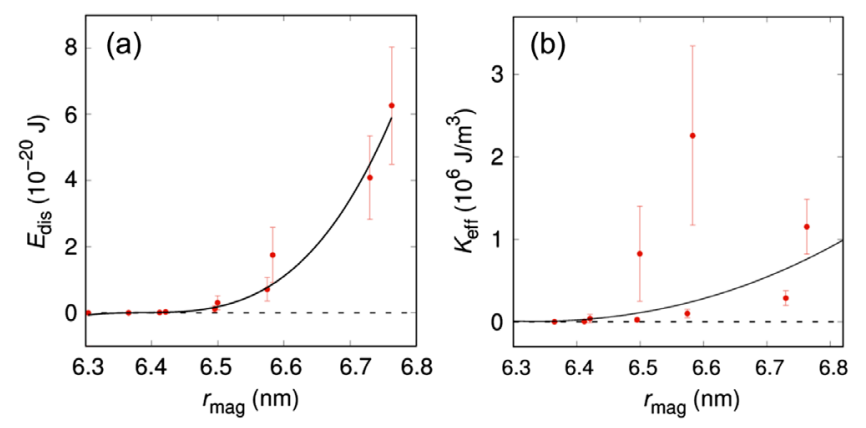

FIG. 7. Dependence of (a) disorder energy $E_{\text {dis }}$ as a function of the coherent magnetic particle radius (black line, spline fit of the data) and (b) the effective disorder energy density $K_{\text {eff }}$ [black line, derivative of spline in (a)].

magnetize toward the surface. We attribute this result to enhanced structural disorder and significantly reduced exchange interaction near the particle surface. A similarly gradual alignment of surface spins has already been discussed by Kodama et al. [7], who find that surface spins can have multiple metastable configurations with the effect that transitions to new equilibrium magnetization states occur with a magnetic field. The magnitude of the surface spin disorder energy shown in Fig. 7(a) increases up to a value of $E_{\mathrm{dis}}(1.2 \mathrm{~T})=6 \times 10^{-20} \mathrm{~J}$. Starting from a negligible magnitude in the spontaneously magnetized particle core $\left(r_{\text {mag }}<6.3 \mathrm{~nm}\right)$, the disorder energy density attains a maximum value of $K_{\text {eff }}=\left(\partial E_{\text {dis }} / \partial V_{\text {mag }}\right) \approx$ $10^{6} \mathrm{~J} \mathrm{~m}^{-3}$ close to the NP surface [Fig. 7(b)]. We note that the obtained maximum effective energy density value exceeds the bulk magnetocrystalline anisotropy $K_{b}=$ $3.6 \times 10^{5} \mathrm{~J} \mathrm{~m}^{-3}$ [62].

The derived energy density is understood as the spatially resolved magnetic disorder anisotropy within the particle. According to phenomenological relations [63], it can be described as a surface anisotropy $K_{S}=K_{\text {eff }} \cdot r_{\text {mag }} / 3$ of the nanoparticle. Recent particle-size-dependent studies indicate that surface anisotropy is not necessarily constant $[48,64]$. Further theoretical studies confirm that the effect of surface anisotropy does not scale with the surface-tovolume ratio but that surface perturbations penetrate to the NP interior transmitted by exchange interactions leading to a reduced coherent magnetic size [8]. Our approach reveals, for the first time experimentally, how the disorder energy anisotropy varies locally within the particle [Fig. 7(b)], an aspect that is not accessible by common integral approaches correlating volume averaged values from different batches of NP sizes. The maximum surface anisotropy of $K_{S} \approx 2.3 \mathrm{~mJ} \mathrm{~m}^{-2}$ is in excellent agreement with Néel surface anisotropy [65] $\left(0.1-1.3 \mathrm{~mJ} \mathrm{~m}^{-2}\right)$, resulting from broken symmetry at the particle surface and concomitant structural relaxation into the particle core, and it is in the order of magnitude of ferromagnetic materials, e.g., $\mathrm{Co}\left(1 \mathrm{~mJ} \mathrm{~m}^{-2}\right), \operatorname{Er}\left(14 \mathrm{~mJ} \mathrm{~m}^{-2}\right), \operatorname{FePt}\left(34 \mathrm{~mJ} \mathrm{~m}^{-2}\right)$, and
$\mathrm{YCo}_{5}\left(34 \mathrm{~mJ} \mathrm{~m}^{-2}\right)$ [52,66]. Ferromagnetic resonance estimates a significantly lower anisotropy for maghemite NPs in ferrofluids $\left(0.03 \mathrm{~mJ} \mathrm{~m}^{-2}\right)$ [67] or for noninteracting $7 \mathrm{~nm}$ maghemite NPs $\left(0.042 \mathrm{~mJ} \mathrm{~m}^{-2}\right)$ [68]. However, these values are in good agreement with the volume averaged disorder anisotropy of our sample of $\left\langle K_{S}\right\rangle=0.096(32) \mathrm{mJ} \mathrm{m}^{-2}$, derived from the maximum disorder energy related to the nuclear particle volume. In this respect, it is noteworthy that the determined values of the surface disorder energies may vary depending on the method and applied magnetic field, as, for instance, a surface anisotropy of $K_{S}=0.027 \mathrm{~mJ} \mathrm{~m}^{-2}$ is obtained from ferromagnetic resonance at $0.1 \mathrm{~T}$ [69].

The gradual decrease of the magnetic disorder parameter (corresponding to enhanced susceptibility) toward the particle interior is likely correlated with reduced structural defect density in the particle core. In addition, spin disorder localized at the particle surface is known to influence the spin configuration in its vicinity via exchange coupling and, thus, to propagate into the particle interior. In this respect, hollow spherical maghemite nanoparticles represent interesting model systems to further investigate surface effects on anisotropy and magnetic disorder [70]. From magnetization measurements for hollow particles, a strength of the surface anisotropy comparable to the results in this study has been observed [71]. Furthermore, based on Monte Carlo simulations, it has been shown that surface spins tend to a disordered state due to the competition between the surface anisotropy and exchange interactions [71].

\section{CONCLUSION}

This work reveals the field dependence of coherent magnetization and magnetic disorder in highly monodisperse cobalt ferrite nanoparticles and elucidates, for the first time experimentally, the intraparticle disorder energy density with spatial resolution. In contrast to the conventional, static picture, the magnetized core size varies significantly with an applied field. This result demonstrates that structural surface disorder is overcome by an increasing magnetic field in order to gradually polarize the surface spins (Fig. 1). Indeed, micromagnetic evaluation establishes fluctuations of magnetocrystalline anisotropy and magnetostrictive contributions as the origins of the observed surface spin disorder, and spin-resolved SANS supports noncorrelated surface spin disorder rather than spin canting at the particle surface. The spin system is characterized by 12 vol\% of spin disorder at the particle surface even at a high magnetic field of $1.2 \mathrm{~T}$. The observed penetration depth of the magnetically perturbed surface region of $0.7 \mathrm{~nm}$ into the nanoparticle interior provides a quantitative insight into the thickness of a magnetic nanoparticle surface. Our in-depth analysis outperforms the traditional macroscopic characterization by revealing the local magnetization response and by providing quantitative evidence for a spatially varying disorder energy in the nanoparticle, 
which is not separable from the bulk magnetocrystalline anisotropy by macroscopic characterization methods. The successive increase of the collinear magnetic nanoparticle volume in a magnetic field discloses that one probes the local energy landscape that is constituted of a disorder energy which increases gradually toward the surface. The effective disorder anisotropy increases up to $K_{\text {eff }} \approx 1 \times$ $10^{6} \mathrm{~J} \mathrm{~m}^{-3}$ close to the particle surface, corresponding to a surface anisotropy of $K_{S} \approx 2.3 \mathrm{~mJ} \mathrm{~m}^{-2}$.

The strength of the presented approach is in the unambiguous separation of surface spin disorder from disorder in the nanoparticle core. It can be employed to reliably understand phenomena such as the particle-size dependence of the surface disorder and the effects of the chemical environment on the surface spins for varying particle coating. By correlating the magnetic surface disorder energy distribution with structural disorder toward the particle surface, the presented approach furthermore provides indirect insight into the defect concentration and depth profile. Looking beyond magnetic applications, such knowledge of the surface morphology of ferrites plays a decisive role in the diffusion-based fields of heterogeneous catalysis and electrochemistry such as solid-state batteries.

\section{ACKNOWLEDGMENTS}

We thank Achim Rosch for fruitful discussion. We further acknowledge Jan Duchoň for HRTEM measurements and EDX maps at Research Centre Rez, Rez near Prague, Czech Republic, and Michael Smik for support during SAXS measurements at JCNS. We thank Annette Schmidt for the opportunity to use the ADE EV7 VSM. We gratefully acknowledge the financial support provided by JCNS to perform the neutron scattering measurements at the Heinz Maier-Leibnitz Zentrum (MLZ), Garching, Germany, and the provision of beam time at the instrument D33 at the Institut Laue-Langevin, Grenoble, France. The open access fee was covered by FILL2030, a European Union project within the European Commission's Horizon 2020 Research and Innovation programme under Grant agreement No. 731096. The presented work was financially supported by the Ministry of Education, Youth and Sport Czech Republic (National Programme of Sustainability II), Project No. LQ1603 (Research for SUSEN). Financial support from the German Research Foundation (DFG Grant No. DI 1788/2-1) as well as the Institutional Strategy of the University of Cologne within the German Excellence Initiative (Max Delbrück-Prize for Young Researchers 2017) are gratefully acknowledged.

\section{APPENDIX A: SYNTHESIS}

Cobalt ferrite NPs are synthesized by thermal decomposition of a mixed $\mathrm{Co}$, Fe-oleate precursor according to Park et al. [43]. The oleate precursors are prepared from the respective metal chlorides and freshly prepared sodium oleate as follows: A solution of sodium oleate is prepared by dissolving $66 \mathrm{mmol}(2.64 \mathrm{~g})$ of $\mathrm{NaOH}$ in a mixture of $10 \mathrm{~mL}$ of $\mathrm{H}_{2} \mathrm{O}$ and $20 \mathrm{~mL}$ of EtOH and dropwise addition of $68 \mathrm{mmol}$ of oleic acid. Water solutions of $15 \mathrm{~mL}$ of $8 \mathrm{mmol}(1.9 \mathrm{~g}) \mathrm{CoCl}_{2} \cdot 6 \mathrm{H}_{2} \mathrm{O}$ and $16 \mathrm{mmol}$ (4.32 g) $\mathrm{FeCl}_{3} \cdot 6 \mathrm{H}_{2} \mathrm{O}$ are added to the prepared sodium oleate solution. $60 \mathrm{~mL}$ of hexane and $10 \mathrm{~mL}$ of EtOH are added to the reaction, and it is refluxed at $60^{\circ} \mathrm{C}$ for $4 \mathrm{~h}$. After the reaction cools down, the oleate complex is washed three times with $50 \mathrm{~mL}$ of water in order to remove $\mathrm{NaCl}$. A brownish viscous mixed oleate complex is obtained by evaporating all solvents including hexane, $\mathrm{EtOH}$, and water. In a second step, the ferrite NPs are synthesized by thermal decomposition of $5 \mathrm{mmol}$ of the prepared oleate precursor with a small amount $(1.6 \mathrm{~mL})$ of additional oleic acid in $25 \mathrm{~mL}$ of octadecene. A heating rate of $2 \mathrm{~K} / \mathrm{min}$ is applied up to the reflux temperature of $315^{\circ} \mathrm{C}$ which is held for a reflux time of $0.5 \mathrm{~h}$. The prepared NPs are precipitated with ethylacetate/EtOH mixture of $1: 1$ for three times and redispersed in toluene.

\section{APPENDIX B: CHARACTERIZATION}

\section{PXRD}

Powder X-ray diffraction (PXRD) is measured with a PANalytical X'Pert PRO diffractometer equipped with $\mathrm{Cu}$ $\mathrm{K}_{\alpha}$ radiation $(\lambda=1.54 \AA$ ), a secondary monochromator, and a PIXcel detector. The sample is measured in the $2 \theta$ range of $5^{\circ}-80^{\circ}$ with a step size of $0.03^{\circ}$. Rietveld refinement is done in FullProf software [72] using a pseudo-Voigt profile function. The instrumental broadening is determined using a $\mathrm{LaB}_{6}$ reference (SR 660b, NIST).

The Rietveld refinement of the PXRD pattern shown in Fig. 8 and in Table I reveals two phases, a spinel ferrite phase and sodium chloride. The presence of sodium chloride in the sample is due to a nonperfect washing

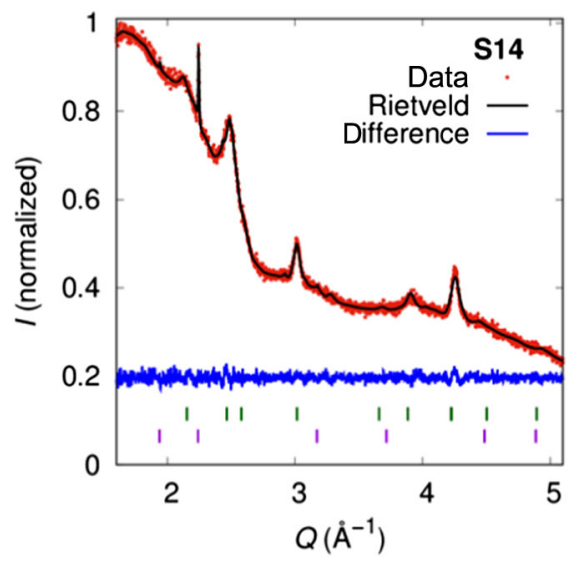

FIG. 8. Rietveld refinement of PXRD pattern of cobalt ferrite NPs. The green and purple vertical lines correspond to the Bragg reflections of spinel cobalt ferrite [73] and of the sodium chloride structure [74], respectively. 
procedure. For the SANS experiment, the preparation is improved by two more purification steps. Nevertheless, this phase does not affect the structural and the magnetic sample properties.

\section{Macroscopic magnetization}

Vibrating sample magnetometry (VSM) measurements are carried out on an ADE EV7 Magnetics vibrating sample magnetometer. $36 \mu \mathrm{l}$ of the dilute NP dispersion is sealed in a Teflon crucible and placed on a glass sample holder. Room-temperature $(298 \mathrm{~K})$ magnetization data are collected in a field range $\pm 1.9 \mathrm{~T}$ with a head drive frequency of $75 \mathrm{~Hz}$. The diamagnetic contribution of the sample holder and solvent is measured independently using a reference measurement of $36 \mu \mathrm{l}$ of toluene.

Additional to the analysis with a single Langevin term (Fig. 5), we also perform an analysis with a distribution $p(\mu)$ of apparent magnetic moments extracted by numerical inversion according to Refs. [49,75]. The extracted distribution of magnetic moments clearly reveals a central peak responsible for the low field magnetization as well as two features with lower moments assigned to the higherfield susceptibility (Fig. 9). The central peak is attributed to the integrated nanoparticle moments and is located in the range of $\mu=1-2 \times 10^{-19} \mathrm{~A} \mathrm{~m}^{2}$ with a maximum at $1.55 \times 10^{-19} \mathrm{~A} \mathrm{~m}^{2}$, corresponding to $1.7 \times 10^{4} \mu_{B}$. This result is in general agreement with our Langevin analysis (Fig. 5) but reveals a moment distribution broader than expected due to a distribution in the magnetic nanoparticle volume and potentially variation of the saturation magnetization [Fig. 9(b)]. The lower moments in the range of $\mu=10^{-21}-10^{-19} \mathrm{~A} \mathrm{~m}^{2}$ are attributed to disordered contributions in the sample.

\section{Mössbauer spectroscopy}

Mössbauer spectroscopy of ${ }^{57} \mathrm{Fe}$ is measured on a Wissel spectrometer using transmission geometry and a proportional detector at ambient temperature without a magnetic field. An $\alpha$-Fe foil is used as standard, and spectra fitting is carried out using the Wissel NORMOS routine [76].

Figure 10 presents a room-temperature Mössbauer spectrogram of the nanoparticles under study. The spectrogram is comparable to Mössbauer spectroscopy by maghemite nanoparticles of similar size [77] close to or above the blocking temperature and is fitted with a singlet and a sextet subspectra including a broad Gaussian distribution due to hyperfine fields or relaxation. We attribute the different subspectra to a distribution of relaxation rates in the nanoparticle sample near the blocking temperature, resulting from the distribution in particle size and, hence, anisotropy energy [78].

The obtained values for the isomer shifts of both subspectra $\left(0.37\right.$ and $0.47 \mathrm{~mm} \mathrm{~s}^{-1}$ for the singlet and sextet, respectively) clearly indicate the exclusive presence of $\mathrm{Fe}^{3+}$ in the sample.

\section{HRTEM and EDX}

HRTEM is done on a JEOL JEM 2200FS operated at $200 \mathrm{kV}$ with a Schottky emitter using bright field mode, scanning transmission mode, energy electron loss spectroscopy, and energy dispersed mapping. The samples are obtained by dropping the toluene dispersion on a coated copper grid.

TABLE I. Rietveld refinement results for the $\mathrm{CoFe}_{2} \mathrm{O}_{4}$ main phase $(F d \overline{3} m)$ with $\mathrm{NaCl}$ impurity $(F m \overline{3} m)$, summarizing the lattice parameters $a$, the oxygen site $u$, the occupancy parameters (not refined), the overall isotropic displacement BOV, and the Lorentzian broadening $Y$.

\begin{tabular}{|c|c|c|c|}
\hline Parameter & $\mathrm{CoFe}_{2} \mathrm{O}_{4}$ & Parameter & $\mathrm{NaCl}$ \\
\hline$a(\AA)$ & $8.362(1)$ & $a(\AA)$ & $5.606(3)$ \\
\hline$u$ & $0.234(4)$ & & \\
\hline $\mathrm{O}(32 \mathrm{e})$ & 4.0 & & \\
\hline Co $(8$ a) & 1.0 & $\mathrm{Na}(4 \mathrm{a})$ & 1.0 \\
\hline $\mathrm{Fe}(16 \mathrm{~d})$ & 2.0 & $\mathrm{Cl}(4 \mathrm{~b})$ & 1.0 \\
\hline $\operatorname{BOV}\left(\AA^{2}\right)$ & $5.9(1)$ & $\mathrm{BOV}\left(\AA^{2}\right)$ & $64(11)$ \\
\hline Profile function & & Thompson-Cox-Hastings Pseudo-Voigt & \\
\hline$Y\left(0.01^{\circ}\right)$ & $0.66(4)$ & & $0.037(6)$ \\
\hline Zero shift $\left(0.01^{\circ}\right)$ & $0.0054(2)$ & & $0.0054(2)$ \\
\hline$R_{f}(\%)$ & 11.4 & & 19.1 \\
\hline$R_{B}(\%)$ & 15.5 & & 11.0 \\
\hline$R_{\mathrm{wp}}(\%)$ & 2.25 & & 2.25 \\
\hline$R_{\exp }(\%)$ & 1.69 & & 1.69 \\
\hline$\chi^{2}$ & 1.77 & & 1.77 \\
\hline Background function & & Interpolation through 30 points & \\
\hline Refined parameters & 5 & & 3 \\
\hline Total fit parameters & & 38 & \\
\hline
\end{tabular}



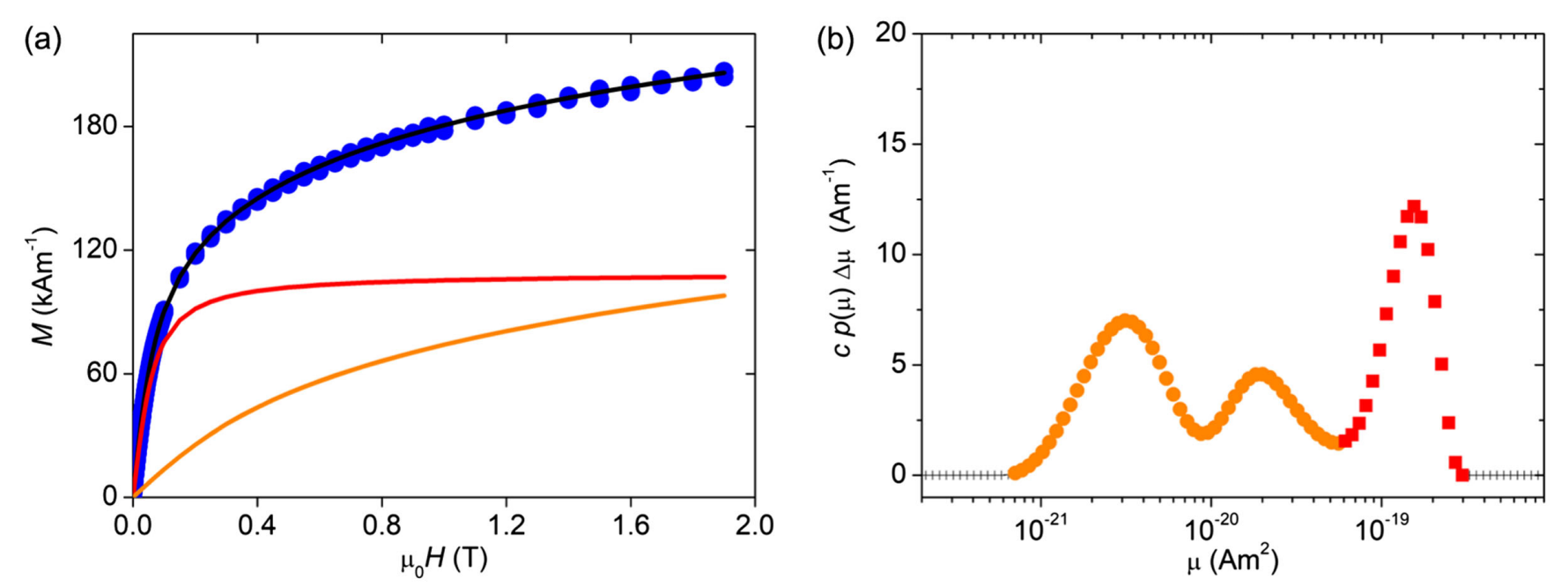

FIG. 9. Macroscopic magnetization measurement analyzed by numerical inversion. (a) VSM data (blue data) and reconstructed data by numerical inversion (black line). The red and orange contributions arise from the extracted large and small magnetic moment distributions, respectively, indicated in (b). $c$ denotes a scaling factor and $\Delta \mu$ the binning width.

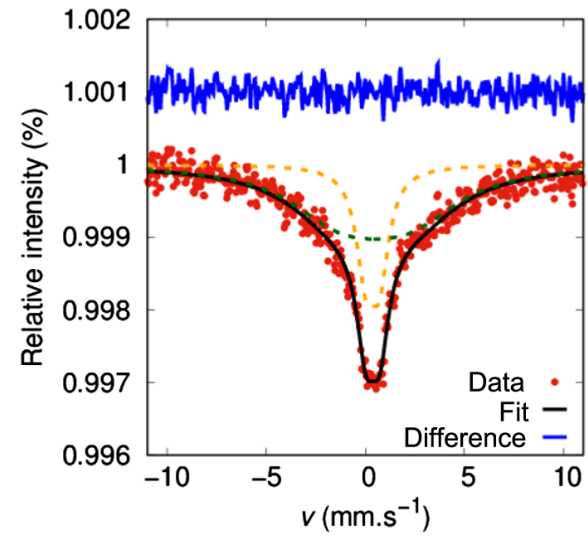

FIG. 10. Room-temperature Mössbauer spectrum. Orange and green dashed lines correspond to the fitted doublet and broad sextet subspectra, respectively.

\section{SAXS}

SAXS measurements are performed at the Gallium Anode Low-Angle X-ray Instrument [79] at JCNS, Forschungszentrum Jülich, Germany. Dilute NP dispersions in toluene $(c=7 \mathrm{mg} / \mathrm{mL})$ are sealed in quartz capillaries and measured using a wavelength of $\lambda=1.3414 \AA$ at two detector distances of 853 and $3548 \mathrm{~mm}$. The data are recorded on a Pilatus $1 \mathrm{M}$ detector, radially averaged, and normalized to absolute units using fluorinated ethylene propylene $1400 \AA(d=0.35 \mathrm{~mm})$ as reference material and toluene background subtraction.

\section{SANSPOL}

SANSPOL is performed at the D33 instrument [80] at ILL, Grenoble, France. Dilute NP dispersions in $d_{8}$-toluene $(c=7 \mathrm{mg} / \mathrm{mL})$ are measured at ambient temperature and under applied horizontal magnetic fields up to $1.2 \mathrm{~T}$ [46].
Two instrument configurations are used with detector distances of 2.5 and $13.4 \mathrm{~m}$ and collimations of 5.3 and $12.8 \mathrm{~m}$, respectively. The incident neutron beam is polarized using a V-shaped supermirror polarizer. The efficiencies of the flipper and supermirror are 0.98 and 0.94, respectively, at a neutron wavelength of $6 \AA$. Data reduction is performed using the GRASP software [81].

The SANSPOL cross section of dilute, noninteracting NPs in a magnetic field applied perpendicular to the neutron beam direction is expressed as [34,82]

$$
\begin{aligned}
I^{ \pm}= & F_{N}^{2}(Q) \mp 2 F_{N}(Q) F_{M}(Q) L(\xi) \sin ^{2} \alpha \\
& +F_{M}^{2}\left[\frac{2 L(\xi)}{\xi}-\sin ^{2} \alpha\left(\frac{3 L(\xi)}{\xi}-1\right)\right],
\end{aligned}
$$

with the azimuthal angle $\alpha$ between the applied magnetic field $\mathbf{H}$ and scattering vector $\mathbf{Q}$, and the Langevin function $L(\xi)$ with $\xi=\mu \mu_{0} H / k_{B} T$, where $k_{B}$ is the Boltzmann constant, $T$ the temperature, $\mu_{0}$ the permeability of the free space, and $\mu$ the integrated particle moment. According to Eq. (B1), the purely nuclear scattering contribution $F_{N}^{2}(Q)$ is accessible from the 2D SANSPOL pattern for $\mathbf{Q} \| \mathbf{H}$ $\left(\sin ^{2} \alpha=0\right)$ and a saturating magnetic field $[L(\xi) / \xi=0]$. The longitudinal magnetic scattering amplitude $F_{M}(Q)$ is accessible via the nuclear-magnetic interference term $I^{+}-I^{-}=-4 F_{N}(Q) F_{M}(Q) L(\xi) \sin ^{2} \alpha$. One can assume that the particle is in a single-domain state for all fields except for a surface region with reduced magnetization; i.e., the magnetization state of the particle core does not change with the field. The integral magnetization is described by Langevin behavior corresponding to the reorientation of the particle moment along the field direction. The magnetic particle moment, increasing with the magnetic field, is given by $\mu(H)=F_{M}(Q=0, H) / b_{H}=V(H) \cdot \rho_{\mathrm{mag}} / b_{H}$ 

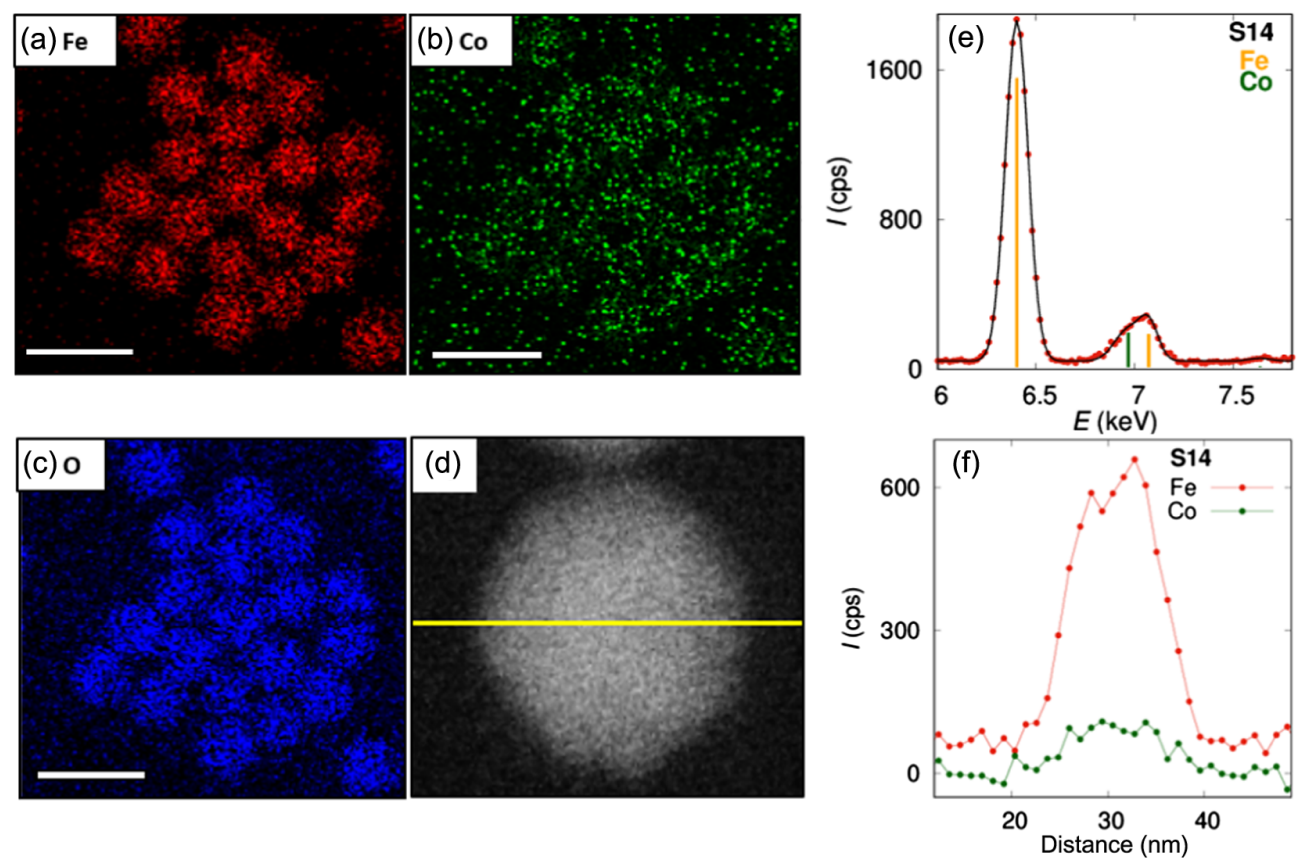

FIG. 11. (a)-(c) EDX map of the distribution of Fe, O, and Co over a region containing 19-20 particles (scale bars of EDX maps, $10 \mathrm{~nm}$ ) with (e) spectra reconstructed from the indicated area. (f) EDX line scan over an arbitrarily selected particle, displayed in (d).

and is used as an input value for the Langevin function $L(\xi)$. The strength of the magnetic scattering is proportional to the magnetic scattering length density $\rho_{\text {mag }}$ that is related to the effective longitudinal magnetization component $M_{z}(H)$ of the core according to

$$
\rho_{\mathrm{mag}}=b_{H} \cdot M_{z}(H)=b_{H} \cdot M_{s} \cdot L(\xi)
$$

where $b_{H}=2.91 \times 10^{8} \mathrm{~A}^{-1} \mathrm{~m}^{-1}$ is the magnetic scattering length. The refined parameters from field-dependent SANSPOL data are summarized in Table II. The difference

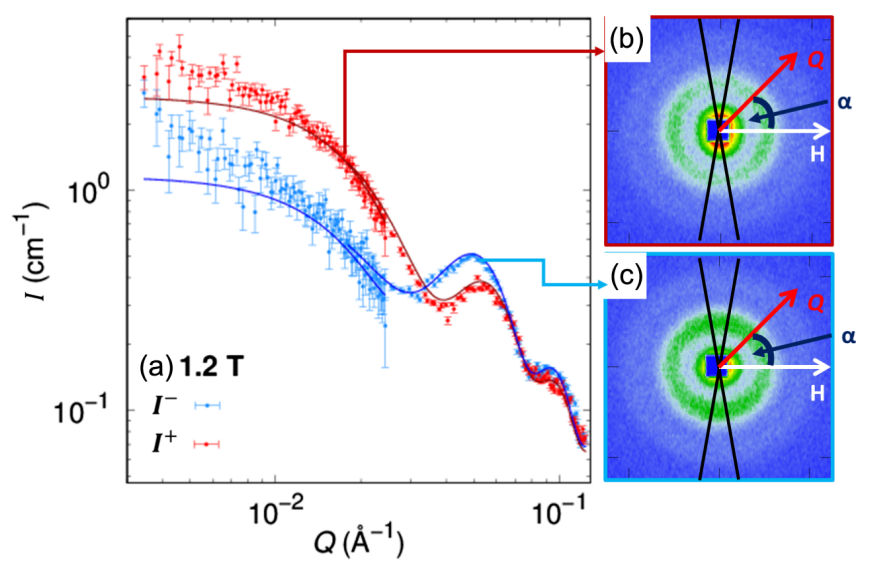

FIG. 12. (a) SANSPOL scattering cross sections $I^{+}$and $I^{-}$ recorded at $1.2 \mathrm{~T}$ along with refinement (full lines). (b),(c) Corresponding 2D scattering patterns showing the $20^{\circ}$ sectors around an angle of $\alpha=90^{\circ}$ between the scattering vector $\mathbf{Q}$ and the applied magnetic field $\mathbf{H}$. method $\left(I^{+}-I^{-}\right)$has the advantage that it eliminates background scattering contributions such as incoherent scattering, potential nonmagnetic contaminations in the sample, or spin-misalignment contributions arising from moments deviating randomly from the field axis. Complementary refinements of the individual $\mathrm{I}^{+}$and $\mathrm{I}^{-}$ cross sections (Fig. 12) confirm the consistency of the results.

\section{NP magnetic morphology: Static vs field-dependent magnetic particle volume}

In order to prove the validity of our nonstatic, fielddependent model of the magnetic form factor, we compare it here with a SANSPOL evaluation based on the commonly used static, field-independent magnetic morphology. In this case, also a core-shell model consisting of a collinearly magnetized particle core and a disordered surface shell is considered. The magnetic core size $r_{\text {mag }}$ is refined in the highest field data (for its best statistics in the nuclearmagnetic interference term) and held constant for all other field-dependent SANS data, leaving the magnetic scattering length density $\rho_{\text {mag }}$ as the only field-dependent fit parameter.

Results of the static model are presented in Fig. 13, and Table III provides a direct comparison of the obtained reduced $\chi^{2}$ for both models. We note that, throughout all datasets, the obtained reduced $\chi^{2}$ is improved for the fielddependent $r_{\text {mag }}$ model by a few percent. In the very low field, the reduced $\chi^{2}$ below unity for both models indicates that the fits are overrated. We attribute this result to the very small magnetic scattering signal in comparison to the 
TABLE II. Parameters refined from field-dependent SANSPOL data, with the magnetic particle radius $r_{\text {mag }}$, the disorder shell thickness $d_{\text {dis }}$, and the magnetic scattering length density $\rho_{\text {mag. }}$ Derived parameters include the magnetic particle volume $V_{\text {mag }}$ and longitudinal magnetization $M_{z}$ as well as average particle magnetization $\langle M\rangle$, considering a nuclear particle volume of $V_{\text {nuc }}=1462(31) \mathrm{nm}^{-3}$ with $r_{\text {nuc }}=7.04(5) \mathrm{nm}$.

\begin{tabular}{lllccrc}
\hline \hline Field $(\mathrm{T})$ & $r_{\text {mag }}(\mathrm{nm})$ & $d_{\text {dis }}(\mathrm{nm})$ & $V_{\operatorname{mag}}\left(\mathrm{nm}^{3}\right)$ & $\rho_{\operatorname{mag}}\left(10^{-7} \AA^{-2}\right)$ & $M_{z}(\mathrm{kA} / \mathrm{m})$ & $\langle M\rangle(\mathrm{kA} / \mathrm{m})$ \\
\hline 0.011 & $6.30(13)$ & $0.74(14)$ & $1047(65)$ & $0.67(7)$ & $23(2)$ & $16(2)$ \\
0.018 & $6.36(14)$ & $0.68(15)$ & $1078(71)$ & $1.33(6)$ & $46(2)$ & $34(3)$ \\
0.028 & $6.41(11)$ & $0.63(12)$ & $1103(57)$ & $1.94(7)$ & $67(2)$ & $50(3)$ \\
0.05 & $6.42(8)$ & $0.62(9)$ & $1108(41)$ & $2.74(7)$ & $94(2)$ & $71(4)$ \\
0.1 & $6.50(6)$ & $0.55(8)$ & $1148(32)$ & $3.67(7)$ & $126(2)$ & $99(4)$ \\
0.2 & $6.49(5)$ & $0.55(7)$ & $1145(26)$ & $4.48(7)$ & $154(2)$ & $121(4)$ \\
0.3 & $6.57(5)$ & $0.47(7)$ & $1188(27)$ & $4.92(8)$ & $169(3)$ & $137(5)$ \\
0.6 & $6.58(4)$ & $0.46(6)$ & $1193(22)$ & $5.82(7)$ & $200(2)$ & $163(5)$ \\
0.88 & $6.73(4)$ & $0.31(6)$ & $1277(23)$ & $5.95(8)$ & $204(3)$ & $179(5)$ \\
1.2 & $6.76(4)$ & $0.28(6)$ & $1294(23)$ & $6.17(7)$ & $212(2)$ & $188(6)$ \\
\hline \hline
\end{tabular}

measurement uncertainty at such a low applied field. However, the field-dependent $r_{\text {mag }}$ model yields a better fit of about $3 \%$ on average (1.05 as compared to 1.08 for the static model) and of 5\%-10\% for intermediate fields $(0.1-0.6 \mathrm{~T})$. This result indicates that the model with variable $r_{\text {mag }}$ improves the fit significantly.

The main effect is directly visible in the comparison to the macroscopic magnetization, where the SANSPOL result $\langle M\rangle$ deviates strongly from the VSM data $\left\langle M_{\mathrm{VSM}}\right\rangle$ as indicated by the red box in Fig. 13(c). Comparison of microscopic SANSPOL results with the independently measured macroscopic magnetization is an important proof of consistency. The deviation shown in Fig. 13(c) is a clear indication that the applied static model is not sufficient to describe the SANSPOL data reliably. In contrast, the fielddependent model yields excellent agreement of microscopic and macroscopic magnetization as shown in Fig. 5.

In consequence, a consistent analysis of our SANSPOL data, in agreement with macroscopic magnetization measurements, is achieved only by consideration of a field-dependent $r_{\text {mag. }}$. This result underlines the need for the spatial sensitivity of magnetic SANS in addition to macroscopic techniques to describe the structural and magnetic details.

\section{POLARIS}

Full polarized small-angle neutron scattering (POLARIS) is done at the KWS-1 instrument [83] operated by Jülich Centre for Neutron Science (JCNS) at Heinz MaierLeibnitz Zentrum (MLZ), Garching, Germany. A dilute noninteracting NP dispersion in $d_{8}$-toluene is measured at ambient temperature and under applied horizontal magnetic fields up to $1.2 \mathrm{~T}$. Measurements are performed at the detector distance of $8 \mathrm{~m}$ with a collimation of $8 \mathrm{~m}$. The incident neutron beam (of $5 \AA$ neutron wavelength) is polarized using a supermirror polarizer, and the polarization of the scattered neutrons is analyzed using a polarized ${ }^{3} \mathrm{He}$ spin filter cell. The incident supermirror gives 0.905 for the wavelength of the experiment with a 0.998 flipper
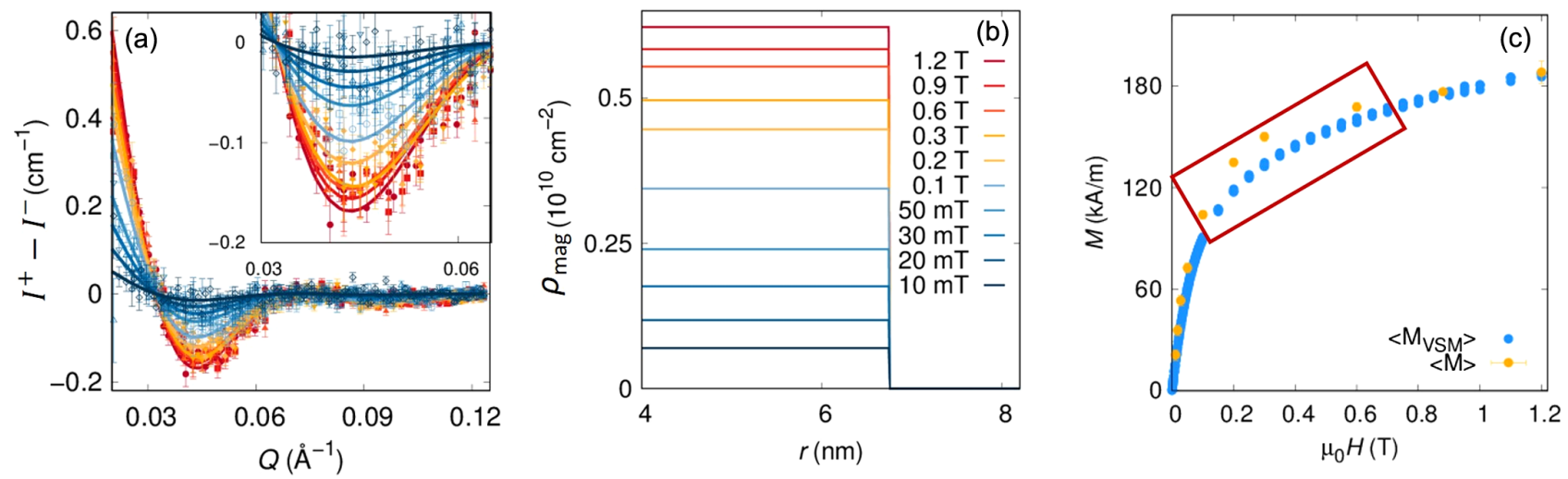

FIG. 13. (a) Nuclear-magnetic scattering interference term $I^{+}-I^{-}$(points) at various applied magnetic fields [same color code as in (b)] and corresponding fits (lines). Inset: Enlarged region of $Q=0.03-0.065 \AA^{-1}$. (b) Field-dependent magnetic scattering length density $\rho_{\text {mag }}$ profiles. (c) Macroscopic longitudinal magnetization $\left\langle M_{\mathrm{VSM}}\right\rangle$ measured at room temperature in comparison with the particle volume averaged magnetization $\langle M\rangle$ as derived from SANSPOL. Deviation in the intermediate field range is indicated (red box). 


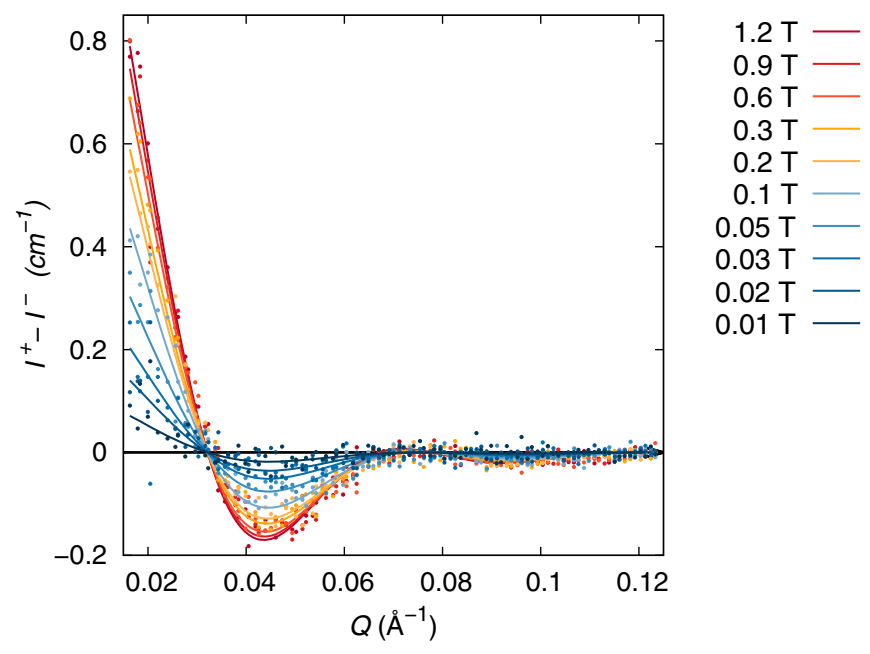

FIG. 14. Fit of the SANSPOL cross term according to micromagnetic theory.

efficiency. The incident beam polarization in this case is slightly reduced by a beam depolarization resulting from the sample slits. At this time, off-line polarized ${ }^{3} \mathrm{He}$ cells are used for KWS-1; therefore, two different cells named Jimmy and Willy with 8.9 and 10.8 bar $\mathrm{cm}$ of ${ }^{3} \mathrm{He}$, respectively, are used [84]. Both cells provide 100 $( \pm 4) \mathrm{h}$ on beam lifetimes. Jimmy and Willy give starting and ending unpolarized neutron transmissions of about 0.21 down to 0.17 and 0.20 going down to 0.14 after a typical half day of use corresponding to initial to final polarization analyzing powers of 0.984 down to 0.976 and 0.995 down to 0.992 for each cell, respectively [83]. Four cell exchanges between the two cells are made during the course of the experiment to maintain good transmission performance. Data reduction and spin-leakage corrections due to polarization inefficiencies as well as solvent subtraction are performed using qtiKWs software [85], and extraction of the azimuthal intensities is carried out using GRASP software [81].

TABLE III. Reduced $\chi^{2}$ values for refinement of the fielddependent SANSPOL data according to either a static or a fielddependent magnetic morphology model. Fit results are shown in Figs. 3 and 13, respectively.

\begin{tabular}{lcc}
\hline \hline Field $(\mathrm{T})$ & $\chi_{\text {red }}^{2}($ static $)$ & $\chi_{\text {red }}^{2}($ field dependent) \\
\hline 1.2 & 1.155 & 1.155 \\
0.88 & 1.356 & 1.341 \\
0.6 & 1.194 & 1.075 \\
0.3 & 1.433 & 1.38 \\
0.2 & 1.121 & 1.088 \\
0.1 & 1.053 & 1.004 \\
0.05 & 1.043 & 1.019 \\
0.028 & 1.015 & 1.007 \\
0.018 & 0.669 & 0.663 \\
0.011 & 0.801 & 0.817 \\
\hline \hline
\end{tabular}

The neutron spin-resolved non-spin-flip $\left(I^{ \pm \pm}\right)$and spinflip $\left(I^{ \pm \mp}\right)$ cross sections of dilute, noninteracting NPs in dispersion are expressed as [34]

$$
\begin{aligned}
& I^{ \pm \pm} \propto F_{N}^{2}+\left|\widetilde{M}_{y}\right|^{2} \sin ^{2} \alpha \cos ^{2} \alpha \mp F_{N} \widetilde{M}_{z} \sin ^{2} \alpha \\
&+\left|\widetilde{M}_{z}\right|^{2} \sin ^{4} \alpha+\text { bgr }, \\
& I^{ \pm \mp} \propto\left|\widetilde{M}_{x}\right|^{2}+\left|\widetilde{M}_{y}\right|^{2} \cos ^{4} \alpha+\left|\widetilde{M}_{z}\right|^{2} \sin ^{2} \alpha \cos ^{2} \alpha+\text { bgr },
\end{aligned}
$$

with $\left|\widetilde{M}_{x}\right|^{2},\left|\widetilde{M}_{y}\right|^{2}$, and $\left|\widetilde{M}_{z}\right|^{2}$ the Fourier transforms of the magnetic correlations along the three Cartesian directions. Terms linear in the transversal component $\widetilde{M}_{y}$ average out in dispersion, since the spin distribution can be assumed to be symmetric around the field direction. Furthermore, due to the particle symmetry, we assume the squared Fourier coefficients of the transversal magnetization to be equal $\left(\widetilde{M}_{x}^{2}=\widetilde{M}_{y}^{2}=\widetilde{M}_{\perp}^{2}\right)$. The non-spin-flip $\left(I^{ \pm \pm}\right)$and spin-flip $\left(I^{ \pm \mp}\right)$ cross sections of dilute, noninteracting NPs in dispersion under an applied magnetic field perpendicular to the neutron beam direction are hence expressed as [34]

$$
\begin{aligned}
& I^{ \pm \pm} \propto F_{N}^{2}+\left|\widetilde{M}_{\perp}\right|^{2} \sin ^{2} \alpha \cos ^{2} \alpha \mp F_{N} \widetilde{M}_{z} \sin ^{2} \alpha \\
&+\left|\widetilde{M}_{z}\right|^{2} \sin ^{4} \alpha+\text { bgr, } \\
& I^{ \pm \mp} \propto\left|\widetilde{M}_{\perp}\right|^{2}\left(1+\cos ^{4} \alpha\right)+\left|\widetilde{M}_{z}\right|^{2} \sin ^{2} \alpha \cos ^{2} \alpha+\mathrm{bgr},
\end{aligned}
$$

with $\left|\widetilde{M}_{\perp}\right|^{2}$ and $\left|\widetilde{M}_{z}\right|^{2}$ the Fourier transforms of the transversal and longitudinal magnetization correlations, respectively, and bgr a scattering background term originating mainly in spin-incoherent scattering contributions.

The non-spin-flip (NSF) and spin-flip (SF) scattering cross sections shown in Figs. 4(a) and 4(b) are radially averaged in the $Q$ range of $0.006-0.016 \AA^{-1}$. The resulting azimuthal SF $\left[\left(I^{+-}+I^{-+}\right) / 2\right]$ and NSF $\left[\left(I^{++}+I^{--}\right) / 2\right]$ cross sections at the measured magnetic field of 1.2 and $0.3 \mathrm{~T}$ are shown in Figs. 4(c)-4(f). The azimuthal SF and NSF intensities show clearly the $\sin ^{2} \alpha \cos ^{2} \alpha$ and $\sin ^{4} \alpha$ anisotropies, respectively, proportional to the longitudinal magnetization $\left|\widetilde{M}_{z}\right|^{2}$. No sign for a $1+\cos ^{4} \alpha$ behavior arising from transversal magnetization correlations is found in the SF data [Figs. 4(e) and 4(f)], and also the NSF data can be described without the need for a transversal magnetization component [Figs. 4(c) and 4(d)]. From SF scattering, the $\left|\widetilde{M}_{z}\right|^{2}$ values of $1.5(1)$ and $1.4(2) \mathrm{cm}^{-1}$ for an applied magnetic field of 1.2 and $0.3 \mathrm{~T}$, respectively, are obtained. Small background values of 0.43(5) and $0.49(7) \mathrm{cm}^{-1}$ are obtained from the fit and are attributed to the spin incoherent scattering from oleic acid at the nanoparticle surface. The obtained $\left|\widetilde{M}_{z}\right|^{2}$ values of $1.59(7)$ and $1.53(1) \mathrm{cm}^{-1}$ from NSF scattering at 1.2 and $0.3 \mathrm{~T}$ are in great agreement with the received values of $\left|\widetilde{M}_{z}\right|^{2}$ values from SF scattering. Background values of 4.82(2) and 
$4.95(2) \mathrm{cm}^{-1}$ at 1.2 and $0.3 \mathrm{~T}$, respectively, in the NSF scattering contribution are assigned to the sum of the spin incoherent and nuclear scattering.

\section{APPENDIX C: MICROMAGNETIC THEORY OF AN INHOMOGENEOUSLY MAGNETIZED PARTICLE}

Based on micromagnetic theory [61], we can derive an analytical expression for the magnetic scattering amplitude under the influence of spatially random magnetocrystalline and magnetostrictive fluctuations:

$$
F_{M}(Q, H)=b_{H}\left[\mu_{0} M_{S} F_{\text {sphere }}\left(Q, r_{\text {nuc }}\right)-g_{H}(Q) p\right] .
$$

The field dependence enters with the dimensionless micromagnetic response function $p=M_{S} /\left[H_{\text {eff }}(Q, H)+\right.$ $\left.2\left\langle H_{K}\right\rangle\right]$ with $\left\langle H_{K}\right\rangle$ the (field-independent) mean magnetocrystalline anisotropy field averaged over the inorganic particle volume. The effective field $H_{\text {eff }}(H, Q)=H(1+$ $\left.l_{H}^{2} Q^{2}\right)$ depends on the applied field $H$ and on the exchange length of the field $l_{H}(H)=\sqrt{2 A /\left(\mu_{0} M_{S} H\right)}$ with the parameter $A$ denoting the exchange stiffness constant. The length scale $l_{H}$ characterizes the range over which perturbations in the magnetization decay.

Equation $(\mathrm{C} 1)$ contains the Fourier coefficient $g_{H}(Q)$, which is independent of the applied magnetic field and contains information on the strength and the spatial structure of perturbing fields associated with the magnetic disorder anisotropy and fluctuations in magnetoelastic coupling. We assume a core-shell morphology for $g_{H}$, with a magnetic core having a reduced or even negligible perturbating disorder field and a surface shell with a drastically increased defect density giving rise to a random site perturbing field and, hence, misalignment of the magnetic moment from the magnetic easy axis of the particle. The exchange interaction is not accessible from the fit due to the restricted $Q$ range.

\section{APPENDIX D: FREE ENERGY CALCULATION}

The field-dependent Zeeman energy $E(H)$ of a nanoparticle in an external field is given by

$E(H)=-\mu \mu_{0} H\langle\cos \gamma\rangle=-\mu_{0} H V_{\mathrm{mag}}(H) M_{z}(H)$,

where $\mu_{0}$ is the permeability of free space and $\mu(H)=$ $V_{\text {mag }}(H) M_{S}$ the integrated particle moment with $V_{\text {mag }}(H)=$ $\frac{4}{3} \pi r_{\text {mag }}^{3}(H)$ the coherently magnetized volume at the magnetic field $H$. The longitudinal magnetization of the coherently magnetized particle core $M_{z}(H)=M_{S}\langle\cos \gamma(H)\rangle$ is directly accessible using polarized SANS [Eq. (B2)]. The Zeeman energy difference between the initial magnetized volume close to remanence and the increased magnetic volume for a specific applied magnetic field amounts to the energy required to align the disordered surface spins.
[1] D. A. Keen and A. L. Goodwin, The Crystallography of Correlated Disorder, Nature (London) 521, 303 (2015).

[2] G. Xu, J. Wen, C. Stock, and P. M. Gehring, Phase Instability Induced by Polar Nanoregions in a Relaxor Ferroelectric System, Nat. Mater. 7, 562 (2008).

[3] S. J. L. Billinge, T. Proffen, V. Petkov, J. L. Sarrao, and S. Kycia, Evidence for Charge Localization in the Ferromagnetic Phase of $\mathrm{La}_{1-x} \mathrm{Ca}_{x} \mathrm{MnO}_{3}$ from High Real-SpaceResolution X-Ray Diffraction, Phys. Rev. B 62, 1203 (2000).

[4] M. Christensen, A. B. Abrahamsen, N. B. Christensen, F. Juranyi, N. H. Andersen, K. Lefmann, J. Andreasson, C. R. H. Bahl, and B. B. Iversen, Avoided Crossing of Rattler Modes in Thermoelectric Materials, Nat. Mater. 7, 811 (2008).

[5] S. Mühlbauer, B. Binz, F. Jonietz, C. Pfleiderer, A. Rosch, A. Neubauer, R. Georgii, and P. Böni, Skyrmion Lattice in a Chiral Magnet, Science 323, 915 (2009).

[6] S. T. Bramwell, S. R. Giblin, S. Calder, R. Aldus, D. Prabhakaran, and T. Fennell, Measurement of the Charge and Current of Magnetic Monopoles in Spin Ice, Nature (London) 461, 956 (2009).

[7] R. H. Kodama, A. E. Berkowitz, E. J. McNiff, and S. Foner, Surface Spin Disorder in $\mathrm{NiFe}_{2} \mathrm{O}_{4}$ Nanoparticles, Phys. Rev. Lett. 77, 394 (1996).

[8] D. A. Garanin and H. Kachkachi, Surface Contribution to the Anisotropy of Magnetic Nanoparticles, Phys. Rev. Lett. 90, 065504 (2003).

[9] F. Luis, J. M. Torres, L. M. Garcia, J. Bartolome, J. Stankiewicz, F. Petroff, F. Fettar, J. L. Maurice, and A. Vaurès, Enhancement of the Magnetic Anisotropy of Nanometer-Sized Co Clusters: Influence of the Surface and of Interparticle Interactions, Phys. Rev. B 65, 094409 (2002).

[10] V. Skumryev, S. Stoyanov, Y. Zhang, G. Hadjipanayis, D. Givord, and J. Nogués, Beating the Superparamagnetic Limit with Exchange Bias, Nature (London) 423, 850 (2003).

[11] G. Salazar-Alvarez, J. Qin, V. Šepelák, I. Bergmann, M. Vasilakaki, K. N. Trohidou, J. D. Ardisson, W. A. Macedo, M. Mikhaylova, M. Muhammed, M. D. Baró, and J. Nogués, Cubic versus Spherical Magnetic Nanoparticles: The Role of Surface Anisotropy, J. Am. Chem. Soc. 130, 13234 (2008).

[12] S. D. Bader, Colloquium: Opportunities in Nanomagnetism, Rev. Mod. Phys. 78, 1 (2006).

[13] Q. A. Pankhurst, J. Connolly, S. K. Jones, and J. Dobson, Applications of Magnetic Nanoparticles in Biomedicine, J. Phys. D 36, R167 (2003).

[14] S. Tong, C. A. Quinto, L. Zhang, P. Mohindra, and G. Bao, Size-Dependent Heating of Magnetic Iron Oxide Nanoparticles, ACS Nano 11, 6808 (2017).

[15] L. Yang, Z. Wang, L. Ma, A. Li, J. Xin, R. Wei, H. Lin, R. Wang, Z. Chen, and J. Gao, The Roles of Morphology on the Relaxation Rates of Magnetic Nanoparticles, ACS Nano 12, 4605 (2018).

[16] C. Niether, S. Faure, A. Bordet, J. Deseure, M. Chatenet, J. Carrey, B. Chaudret, and A. Rouet, Improved Water Electrolysis Using Magnetic Heating of $\mathrm{FeC}-\mathrm{Ni}$ Core-shell Nanoparticles, Nat. Energy 3, 476 (2018). 
[17] W. Li, Z. Liang, Z. Lu, X. Tao, K. Liu, H. Yao, and Y. Cui, Magnetic Field-Controlled Lithium Polysulfide Semiliquid Battery with Ferrofluidic Properties, Nano Lett. 15, 7394 (2015).

[18] P. Bender, J. Fock, C. Frandsen, M. F. Hansen, C. Balceris, F. Ludwig, O. Posth, E. Wetterskog, L. K. Bogart, P. Southern, W. Szczerba, L. Zeng, K. Witte, C. Grüttner, F. Westphal, D. Honecker, D. González-Alonso, L. Fernández Barquín, and C. Johansson, Relating Magnetic Properties and High Hyperthermia Performance of Iron Oxide Nanoflowers, J. Phys. Chem. C 122, 3068 (2018).

[19] A. Lak, M. Cassani, B. T. Mai, N. Winckelmans, D. Cabrera, E. Sadrollahi, S. Marras, H. Remmer, S. Fiorito, L. Cremades-Jimeno, F. J. Litterst, F. Ludwig, L. Manna, F. J. Teran, S. Bals, and T. Pellegrino, $\mathrm{Fe}^{2+}$ Deficiencies, $\mathrm{FeO}$ Subdomains, and Structural Defects Favor Magnetic Hyperthermia Performance of Iron Oxide Nanocubes into Intracellular Environment, Nano Lett. 18, 6856 (2018).

[20] A. Lappas, G. Antonaropoulos, K. Brintakis, M. Vasilakaki, K. N. Trohidou, V. Iannotti, G. Ausanio, A. Kostopoulou, M. Abeykoon, I. K. Robinson, and E. S. Bozin, VacancyDriven Noncubic Local Structure and Magnetic Anisotropy Tailoring in $\mathrm{Fe}_{x} \mathrm{O}-\mathrm{Fe}_{3-\delta} \mathrm{O}_{4}$ Nanocrystals, Phys. Rev. X 9 , 041044 (2019).

[21] B. N. Pianciola, E. Lima, H. E. Troiani, L. C. Nagamine, R. Cohen, and R. D. Zysler, Size and Surface Effects in the Magnetic Order of $\mathrm{CoFe}_{2} \mathrm{O}_{4}$ Nanoparticles, J. Magn. Magn. Mater. 377, 44 (2015).

[22] V. Bonanni, M. Basini, D. Peddis, A. Lascialfari, G. Rossi, and P. Torelli, X-Ray Magnetic Circular Dichroism Discloses Surface Spins Correlation in Maghemite Hollow Nanoparticles, Appl. Phys. Lett. 112, 022404 (2018).

[23] D. S. Negi, H. Sharona, U. Bhat, S. Palchoudhury, A. Gupta, and R. Datta, Surface Spin Canting in $\mathrm{Fe}_{3} \mathrm{O}_{4}$ and $\mathrm{CoFe}_{2} \mathrm{O}_{4}$ Nanoparticles Probed by High-Resolution Electron Energy Loss Spectroscopy, Phys. Rev. B 95, 174444 (2017).

[24] M. Darbandi, F. Stromberg, J. Landers, N. Reckers, B. Sanyal, W. Keune, and H. Wende, Nanoscale Size Effect on Surface Spin Canting in Iron Oxide Nanoparticles Synthesized by the Microemulsion Method, J. Phys. D 45, 195001 (2012).

[25] D. Peddis, M. V. Mansilla, S. Mørup, C. Cannas, A. Musinu, G. Piccaluga, F. D’Orazio, F. Lucari, and D. Fiorani, SpinCanting and Magnetic Anisotropy in Ultrasmall $\mathrm{CoFe}_{2} \mathrm{O}_{4}$ Nanoparticles, J. Phys. Chem. B 112, 8507 (2008).

[26] J. A. De Toro, M. Vasilakaki, S. S. Lee, M. S. Andersson, P. S. Normile, N. Yaacoub, P. Murray, E. H. Sánchez, P. Muńiz, D. Peddis, R. Mathieu, K. Liu, J. Geshev, K. N. Trohidou, and J. Nogués, Remanence Plots as a Probe of Spin Disorder in Magnetic Nanoparticles, Chem. Mater. 29, 8258 (2017).

[27] L. Kwan, J. Jung-tak, N. Hiroshi, N. Shigeki, S. H. Paek, and B. Seongtae, External Magnetic Field Dependent Shift of Superparamagnetic Blocking Temperature due to Corel Surface Disordered Spin Interactions, Nanotechnology 28, 075710 (2017).

[28] E. Winkler, R. D. Zysler, M. V. Mansilla, and D. Fiorani, Surface Anisotropy Effects in NiO Nanoparticles, Phys. Rev. B 72, 132409 (2005).
[29] M. P. Morales, S. Veintemillas-Verdaguer, M. I. Montero, C. J. Serna, A. Roig, L. Casas, B. Martínez, and F. Sandiumenge, Surface and Internal Spin Canting in $\gamma-\mathrm{Fe}_{2} \mathrm{O}_{3}$ Nanoparticles, Chem. Mater. 11, 3058 (1999).

[30] Z. Nedelkoski, D. Kepaptsoglou, L. Lari, T. Wen, R. A. Booth, S. D. Oberdick, P. L. Galindo, Q. M. Ramasse, R. F. L. Evans, S. Majetich, and V. K. Lazarov, Origin of Reduced Magnetization and Domain Formation in Small Magnetite Nanoparticles, Sci. Rep. 7, 45997 (2017).

[31] B. Bittová, J. P. Vejpravová, M. Kalbáć, S. Burianová, A. Mantliková, S. Daniš, and S. Doyle, Magnetic Properties of Iron Catalyst Particles in Hipco Single Wall Carbon Nanotubes, J. Phys. Chem. C 115, 17303 (2011).

[32] S. Kubičková, D. Nižňanský, M. P. M. Herrero, G. Salas, and J. Vejpravová, Structural Disorder versus Spin Canting in Monodisperse Maghemite Nanocrystals, Appl. Phys. Lett. 104, 223105 (2014).

[33] E. Wetterskog, C.-W. Tai, J. Grins, L. Bergström, and G. Salazar-Alvarez, Anomalous Magnetic Properties of Nanoparticles Arising from Defect Structures: Topotaxial Oxidation of $\mathrm{Fe}_{1-x} \mathrm{O} \mid \mathrm{Fe}_{3-\delta} \mathrm{O}_{4}$ Core-Shell Nanocubes to Single-Phase Particles, ACS Nano 7, 7132 (2013).

[34] S. Mühlbauer, D. Honecker, E. A. Périgo, F. Bergner, S. Disch, A. Heinemann, S. Erokhin, D. Berkov, C. Leighton, M. R. Eskildsen, and A. Michels, Magnetic SmallAngle Neutron Scattering, Rev. Mod. Phys. 91, 015004 (2019).

[35] S. Disch, E. Wetterskog, R. P. Hermann, A. Wiedenmann, U. Vainio, G. Salazar-Alvarez, L. Bergström, and T. Brückel, Quantitative Spatial Magnetization Distribution in Iron Oxide Nanocubes and Nanospheres by Polarized Small-Angle Neutron Scattering, New J. Phys. 14, 013025 (2012).

[36] K. L. Krycka, R. A. Booth, C. R. Hogg, Y. Ijiri, J. A. Borchers, W. C. Chen, S. M. Watson, M. Laver, T. R. Gentile, L. R. Dedon, S. Harris, J. J. Rhyne, and S. A. Majetich, Core-Shell Magnetic Morphology of Structurally Uniform Magnetite Nanoparticles, Phys. Rev. Lett. 104, 207203 (2010).

[37] K. L. Krycka, J. A. Borchers, R. A. Booth, S. A. Majetich, Y. Ijiri, K. Hasz, and J. J. Rhyne, Origin of Surface Canting within $\mathrm{Fe}_{3} \mathrm{O}_{4}$ Nanoparticles, Phys. Rev. Lett. 113, 147203 (2014).

[38] K. Hasz, Y. Ijiri, K. L. Krycka, J. A. Borchers, R. A. Booth, S. Oberdick, and S. A. Majetich, Particle Moment Canting in $\mathrm{CoFe}_{2} \mathrm{O}_{4}$ Nanoparticles, Phys. Rev. B 90, 180405(R) (2014).

[39] L. G. Vivas, R. Yanes, and A. Michels, Small-Angle Neutron Scattering Modeling of Spin Disorder in Nanoparticles, Sci. Rep. 7, 13060 (2017).

[40] M. Bersweiler, P. Bender, L. G. Vivas, M. Albino, M. Petrecca, S. Mühlbauer, S. Erokhin, D. Berkov, C. Sangregorio, and A. Michels, Size-Dependent Spatial Magnetization Profile of Manganese-Zinc Ferrite $\mathrm{Mn}_{0.2} \mathrm{Zn}_{0.2} \mathrm{Fe}_{2.6} \mathrm{O}_{4}$ Nanoparticles, Phys. Rev. B 100, 144434 (2019).

[41] S. D. Oberdick, A. Abdelgawad, C. Moya, S. MesbahiVasey, D. Kepaptsoglou, V. K. Lazarov, R. F. Evans, D. Meilak, E. Skoropata, J. Van Lierop, I. Hunt-Isaak, H. Pan, 
Y. Ijiri, K. L. Krycka, J. A. Borchers, and S. A. Majetich, Spin Canting across Core/Shell $\mathrm{Fe}_{3} \mathrm{O}_{4} / \mathrm{Mn}_{x} \mathrm{Fe}_{3-x} \mathrm{O}_{4}$ Nanoparticles, Sci. Rep. 8, 3425 (2018).

[42] A. Bhattacharjee and P. Gütlich, Mössbauer Spectroscopic Study of Low-Temperature Spin Structure and Magnetic Interactions in $\left\{\mathrm{N}\left(\mathrm{n}-\mathrm{C}_{5} \mathrm{H}_{11}\right)_{4}\left[\mathrm{MnIIFeIII}\left(\mathrm{C}_{2} \mathrm{O}_{4}\right)_{3}\right]\right\} \infty$, J. Phys. Condens. Matter 19, 356201 (2007).

[43] J. Park, K. An, Y. Hwang, J.-G. Park, H.-J. Noh, J.-Y. Kim, J.-H. Park, N.-M. Hwang, and T. Hyeon, Ultra-Large-Scale Syntheses of Monodisperse Nanocrystals, Nat. Mater. 3, 891 (2004).

[44] D. Zákutná, D. Honecker, and S. Disch, Phase-Transfer and Stabilization of Highly Monodisperse Ferrite Nanoparticles into Polar Solvents by Ligand Exchange Synthesis, J. Nanosci. Nanotechnol. 19, 5048 (2019).

[45] A. I. Frenkel, C. W. Hills, and R. G. Nuzzo, A View from the Inside: Complexity in the Atomic Scale Ordering of Supported Metal Nanoparticles, J. Phys. Chem. B 105, 12689 (2001).

[46] D. Honecker, S. Disch, D. Hoffelner, and D. Zákutná, Polarised SANS Measurements on Hybrid Ferrofluidic dispersions. Institut Laue-Langevin (ILL), 2015, https:// doi.org/10.5291/ILL-DATA.5-53-261.

[47] A. R. Denton and N. W. Ashcroft, Vegard's Law, Phys. Rev. A 43, 3161 (1991).

[48] S. Singh, K. L. Pisane, and M. S. Seehra, A Core-ShellSurface Layer Model to Explain the Size Dependence of Effective Magnetic Anisotropy in Magnetic Nanoparticles, in Proceedings of the 2017 IEEE 17th International Conference on Nanotechnology (IEEE-NANO) (IEEE, New York, 2017), pp. 1014-1018.

[49] P. Bender, C. Balceris, F. Ludwig, O. Posth, L. K. Bogart, W. Szczerba, A. Castro, L. Nilsson, R. Costo, H. Gavilán, D. González-Alonso, I. D. Pedro, L. F. Barquin, and C. Johansson, Distribution Functions of Magnetic Nanoparticles Determined by a Numerical Inversion Method, New J. Phys. 19, 073012 (2017).

[50] I. V. Golosovsky, M. Tovar, U. Hoffman, I. Mirebeau, F. Fauth, D. A. Kurdyukov, and Y. A. Kumzerov, Diffraction Studies of the Crystalline and Magnetic Structures of $\gamma-\mathrm{Fe}_{2} \mathrm{O}_{3}$ Iron Oxide Nanostructured in Porous Glass, J. Exp. Theor. Phys. Lett. 83, 298 (2006).

[51] Y. Labaye, O. Crisan, L. Berger, J. M. Greneche, and J. M. D. Coey, Surface Anisotropy in Ferromagnetic Nanoparticles, J. Appl. Phys. 91, 8715 (2002).

[52] L. Berger, Y. Labaye, M. Tamine, and J. M. D. Coey, Ferromagnetic Nanoparticles with Strong Surface Anisotropy: Spin Structures and Magnetization Processes, Phys. Rev. B 77, 104431 (2008).

[53] P. D. Thang, G. Rijnders, and D. H. A. Blank, Spinel Cobalt Ferrite by Complexometric Synthesis, J. Magn. Magn. Mater. 295, 251 (2005).

[54] A. E. Clark and E. Callen, Néel Ferrimagnets in Large Magnetic Fields, J. Appl. Phys. 39, 5972 (1968).

[55] P. Bender, L. K. Bogart, O. Posth, W. Szczerba, S. E. Rogers, A. Castro, L. Nilsson, L. J. Zeng, A. Sugunan, J. Sommertune, A. Fornara, D. González-Alonso, L. F. Barquín, and C. Johansson, Structural and Magnetic Properties of Multi-core Nanoparticles Analysed Using a Generalised Numerical Inversion Method, Sci. Rep. 7, 45990 (2017).
[56] D. V. Berkov, P. Görnert, N. Buske, C. Gansau, J. Mueller, M. Giersig, W. Neumann, and D. Su, New Method for the Determination of the Particle Magnetic Moment Distribution in a Ferrofluid, J. Phys. D 33, 331 (2000).

[57] J. Van Rijssel, B. W. Kuipers, and B. H. Erné, Nonregularized Inversion Method from Light Scattering Applied to Ferrofluid Magnetization Curves for Magnetic Size Distribution Analysis, J. Magn. Magn. Mater. 353, 110 (2014).

[58] D. Schmidt, D. Eberbeck, U. Steinhoff, and F. Wiekhorst, Finding the Magnetic Size Distribution of Magnetic Nanoparticles from Magnetization Measurements via the Iterative Kaczmarz Algorithm, J. Magn. Magn. Mater. 431, 33 (2017).

[59] T. E. Torres, E. Lima, A. Mayoral, A. Ibarra, C. Marquina, M. R. Ibarra, and G. F. Goya, Validity of the Néel-Arrhenius Model for Highly Anisotropic $\mathrm{Co}_{x} \mathrm{Fe}_{3-x} \mathrm{O}_{4}$ Nanoparticles, J. Appl. Phys. 118, 183902 (2015).

[60] I. C. Nlebedim, J. E. Snyder, A. J. Moses, and D. C. Jiles, Effect of Deviation from Stoichiometric Composition on Structural and Magnetic Properties of Cobalt Ferrite, $\mathrm{Co}_{x} \mathrm{Fe}_{3-x} \mathrm{O}_{4}(x=0.2$ to 1.0), J. Appl. Phys. 111, $07 \mathrm{D} 704$ (2012).

[61] H. Kronmüller and M. Fähnle, Micromagnetic Theory of Phase Transitions in Inhomogeneous Ferromagnets. I. Basic Equations, Phys. Status Solidi (b) 97, 513 (1980).

[62] H. Shenker, Magnetic Anisotropy of Cobalt Ferrite $\left(\mathrm{Co}_{1.01} \mathrm{Fe}_{2.00} \mathrm{O}_{3.62}\right)$ and Nickel Cobalt Ferrite $\left(\mathrm{Ni}_{0.72} \mathrm{Fe}_{0.20} \mathrm{Co}_{0.08} \mathrm{Fe}_{2} \mathrm{O}_{4}\right)$, Phys. Rev. 107, 1246 (1957).

[63] R. Yanes, O. Chubykalo-Fesenko, H. Kachkachi, D. A. Garanin, R. Evans, and R. W. Chantrell, Effective Anisotropies and Energy Barriers of Magnetic Nanoparticles with Néel Surface Anisotropy, Phys. Rev. B 76, 064416 (2007).

[64] K. L. Pisane, S. Singh, and M. S. Seehra, Unusual Enhancement of Effective Magnetic Anisotropy with Decreasing Particle Size in Maghemite Nanoparticles, Appl. Phys. Lett. 110, 222409 (2017).

[65] L. Néel, Anisotropie Magnetique Superficielle et Surstructures d'Orientation, J. Phys. Radium 15, 225 (1954).

[66] P. Gambardella, S. Rusponi, M. Veronese, S. S. Dhesi, C. Grazioli, A. Dallmeyer, I. Cabria, R. Zeller, P. H. Dederichs, K. Kern, C. Carbone, and H. Brune, Giant Magnetic Anisotropy of Single Cobalt Atoms and Nanoparticles, Science 300, 1130 (2003).

[67] Y. L. Raikher and R. Perzynski, Ferromagnetic Resonance in Nanoparticles with Surface Pinning, J. Magn. Magn. Mater. 272-276, E1219 (2004).

[68] J. L. Dormann, F. D’Orazio, F. Lucari, E. Tronc, P. Prené, J. P. Jolivet, D. Fiorani, R. Cherkaoui, and M. Noguès, Thermal Variation of the Relaxation Time of the Magnetic Moment of $\gamma-\mathrm{Fe}_{2} \mathrm{O}_{3}$ Nanoparticles with Interparticle Interactions of Various Strengths, Phys. Rev. B 53, 14291 (1996).

[69] F. Gazeau, J. C. Bacri, F. Gendron, R. Perzynski, Y. L. Raikher, V. I. Stepanov, and E. Dubois, Magnetic Resonance of Ferrite Nanoparticles: Evidence of Surface Effects, J. Magn. Magn. Mater. 186, 175 (1998).

[70] F. Sayed, N. Yaacoub, Y. Labaye, R. S. Hassan, G. Singh, P. A. Kumar, J. M. Greneche, R. Mathieu, G. C. Hadjipanayis, E. Agostinelli, and D. Peddis, Surface Effects in Ultrathin Iron Oxide Hollow Nanoparticles: Exploring Magnetic Disorder at the Nanoscale, J. Phys. Chem. C 122, 7516 (2018). 
[71] A. Cabot, A. P. Alivisatos, V. F. Puntes, L. Balcells, O. Iglesias, and A. Labarta, Magnetic Domains and Surface Effects in Hollow Maghemite Nanoparticles, Phys. Rev. B 79, 094419 (2009).

[72] J. Rodríguez-Carvajal, Recent Advances in Magnetic Structure Determination by Neutron Powder Diffraction, Physica (Amsterdam) 192B, 55 (1993).

[73] G. Natta and L. Passerini, Spinelli del cobalto bivalenti, aluminato, cromito, ferrito e cobaltito cobaltosi, Gazz. Chim. Ital. 59, 280 (1929).

[74] J. D. Hanawalt, H. W. Rinn, and L. K. Frevel, Chemical Analysis by X-Ray Diffraction, Ind. Eng. Chem., Anal. Ed. 10, 457 (1938).

[75] P. Bender, A Collection of Python Programs for the Analysis of Magnetization and Scattering Data of Magnetic Nanoparticles, https://github.com/PBenderLux/Data-analysis.

[76] R. A. Brand, NORMOS, http://physics-astronomy-manuals .wwu.edu/Wissel\%20NORMOS\%2090\%20Manual.pdf.

[77] L. Rebbouh, R. P. Hermann, F. Grandjean, T. Hyeon, K. An, A. Amato, and G. J. Long, ${ }^{57} \mathrm{Fe}$ Mossbauer Spectral and Muon Spin Relaxation Study of the Magnetodynamics of Monodispersed $\gamma-\mathrm{Fe}_{2} \mathrm{O}_{3}$ Nanoparticles, Phys. Rev. B 76, 174422 (2007).

[78] E. Tronc, A. Ezzir, R. Cherkaoui, C. Chanéac, M. Noguès, H. Kachkachi, D. Fiorani, A. M. Testa, J. M. Grenèche, and
J. P. Jolivet, Surface-Related Properties of $\gamma-\mathrm{Fe}_{2} \mathrm{O}_{3}$ Nanoparticles, J. Magn. Magn. Mater. 221, 63 (2000).

[79] E. Kentzinger, M. Krutyeva, and U. Rücker, GALAXI: Gallium Anode Low-Angle X-Ray Instrument, J. LargeScale Res. Facil. 2, A61 (2016).

[80] C. D. Dewhurst, I. Grillo, D. Honecker, M. Bonnaud, M. Jacques, C. Amrouni, A. Perillo-Marcone, G. Manzin, and R. Cubitt, The Small-Angle Neutron Scattering Instrument D33 at the Institut Laue-Langevin, J. Appl. Crystallogr. 49, 1 (2016).

[81] C. D. Dewhurst, Graphical Reduction and Analysis SANS Program for Matlab, https://www.ill.eu/users/support-labsinfrastructure/software-scientific-tools/grasp/.

[82] A. Wiedenmann, Polarized SANS for Probing Magnetic Nanostructures, Physica (Amsterdam) 356B, 246 (2005).

[83] A. V. Feoktystov, H. Frielinghaus, Z. Di, S. Jaksch, V. Pipich, M.-S. Appavou, E. Babcock, R. Hanslik, R. Engels, G. Kemmerling, H. Kleines, A. Ioffe, D. Richter, and T. Brückel, KWS-1 High-Resolution Small-Angle Neutron Scattering Instrument at JCNS: Current State, J. Appl. Crystallogr. 48, 61 (2015).

[84] Z. Salhi, E. Babcock, P. Pistel, and A. Ioffe, ${ }^{3} \mathrm{He}$ Neutron Spin Filter Cell Development Program at JCNS, J. Phys. Conf. Ser. 528, 012015 (2014).

[85] V. Pipich, QтіKWS, https://www.qtikws.de. 